\title{
Supporting Information for Chiral Brønsted Acid-Catalyzed Tandem Aza-Ene Type Reaction/Cyclization Cascade for a One-Pot Entry to Enantioenriched Piperidines
}

\author{
Masahiro Terada*, Kyoko Machioka, and Keiichi Sorimachi \\ Graduate School of Science, Department of Chemistry, Tohoku University, Sendai 980-8578, Japan.
}

General Information: Infrared spectra were recorded on a Shimazu FTIR-8600PC spectrometer. Optical rotations were measured on a Jasco DIP-1000 digital polarimeter with a sodium lamp and reported as follows; $[\alpha]_{\lambda}{ }^{\mathrm{T}}{ }^{\circ} \mathrm{C}$ ( $c=\mathrm{g} / 100 \mathrm{~mL}$, solvent). $\quad{ }^{1} \mathrm{H}$ NMR spectra were recorded on the BRUKER AVANCE-600 $(600 \mathrm{MHz})$ spectrometer or JEOL GSX-270 (270 MHz) spectrometer. Chemical shifts are reported in ppm from the solvent resonance as the internal standard $\left(\mathrm{CDCl}_{3}: 7.26 \mathrm{ppm},\left(\mathrm{CD}_{3}\right)_{2} \mathrm{CO}: 2.09 \mathrm{ppm}\right)$. Data are reported as follows: chemical shift, integration, multiplicity ( $\mathrm{s}=$ singlet, $\mathrm{d}=$ doublet, $\mathrm{t}=$ triplet, $\mathrm{q}=$ quartet, $\mathrm{br}=$ broad, $\mathrm{m}=$ multiplet $)$, and coupling constants $(\mathrm{Hz})$.

${ }^{13} \mathrm{C}$ NMR spectra were recorded on the BRUKER AVANCE-600 (150.9 MHz) spectrometer or JEOL GSX-270 (67.8 $\mathrm{MHz}$ ) spectrometer with complete proton decoupling. Chemical shifts are reported in ppm from the solvent resonance as the internal standard $\left(\mathrm{CDCl}_{3}: 77.0 \mathrm{ppm},\left(\mathrm{CD}_{3}\right)_{2} \mathrm{CO}: 29.8 \mathrm{ppm}\right)$. Analytical thin layer chromatography (TLC) was performed on Merck precoated TLC plates (silica gel $60 \mathrm{GF}_{254}, 0.25 \mathrm{~mm}$ ). Flash column chromatography was performed on silica gel 60N (spherical, neutral, 100-210 $\mu \mathrm{m}$; Kanto Chemical Co., Inc.). Mass spectra analysis was performed on a Bruker Daltonics APEX III FT-ICR-MS spectrometer at the Instrumental Analysis Center for Chemistry, Graduate School of Science, Tohoku University.

All reactions were carried out under a nitrogen $\left(\mathrm{N}_{2}\right)$ atmosphere in dried glassware. All substrates were purified by column chromatography or distillation prior to use. Dichloromethane, toluene and tetrahydrofuran were supplied from Kanto Chemical Co., Inc. as "Dehydrated solvent system". 1,2-Dichloroethane was dried over activated MS4A and used under $\mathrm{N}_{2}$ atomosphere. Imines $\left(\mathbf{1} \mathbf{a}-\mathbf{d}^{1}, \mathbf{e}^{2}, \mathbf{f}^{3}, \mathbf{g}^{4}\right)$ and enecarbamate $\left(\mathbf{5 a}^{5}\right)$ were prepared according to the literature procedure. The other simple chemicals were purchased and used as such.

(1) Wenzel, A. G.; Jacobsen, E. N. J. Am. Chem. Soc. 2002, 124, 12964.

(2) Okada, A.; Shibuguchi, T.; Ohshima, T.; Masu, H.; Yamaguchi, K.; Shibasaki, M. Angew. Chem., Int. Ed. 2005, $44,4564$.

(3) Nakamura, Y.; Matsubara, R.; Kiyohara, H.; Kobayashi, S. Org. Lett. 2003, 5, 2481.

(4) Song, J.; Wang, Y.; Deng, L. J. Am. Chem. Soc. 2006, 128, 6048.

(5) Wieber, G. M.; Hegedus, L. S.; Akermark, B.; Michalson, E. T. J. Org. Chem. 1989, 54, 4649. 


\section{Experimental Section}

\section{Tandem Aza-Ene Type Reaction/Cyclization Catalyzed by Chiral Brønsted Acid $((R)-3 d)$}

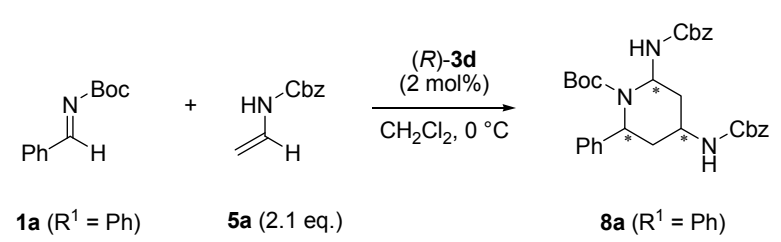

Representative Procedure for the Tandem Aza-Ene Type Reaction/Cyclization of Enecarbamate 5a with tert-Butyl benzylidenecarbamate 1a $\left(\mathbf{R}^{1}=\right.$ Ph) Catalyzed by Chiral Bronsted Acid $(R)-3 d(2$ mol\%): To a dried test tube was weighted chiral phosphoric acid $(R)-\mathbf{3 d}(1.31 \mathrm{mg}, 0.002 \mathrm{mmol})$ and imine $\mathbf{1 a}\left(\mathrm{R}^{1}=\mathrm{Ph}\right)(20.5 \mathrm{mg}, 0.10$ mmol). The atmosphere was replaced by nitrogen. After catalyst $(R)-\mathbf{3 d}$ and imine $\mathbf{1 a}\left(\mathrm{R}^{1}=\mathrm{Ph}\right)$ were dissolved in dichloromethane $(1.0 \mathrm{~mL})$, enecarbamate $5 \mathbf{a}(37.2 \mathrm{mg}, 0.21 \mathrm{mmol})$ was introduced at $0{ }^{\circ} \mathrm{C}$. The reaction mixture was stirred for $1 \mathrm{~h}$. The reaction was quenched by addition of saturated aqueous $\mathrm{NaHCO}_{3}$, and then the resulting mixture was extracted with dichloromethane and dried over anhydrous $\mathrm{Na}_{2} \mathrm{SO}_{4}$. After the solvents were evaporated, the crude material was purified by chromatography (hexane/EtOAc $=8 / 1-1 / 1$ as eluent) to give piperidine derivatives 8a $\left(\mathrm{R}^{1}=\mathrm{Ph}\right)$ in $99 \%$ yield as a white solid.<smiles>O=C(NCCCCCCOC(=O)OCc1ccccc1)NC(=O)c1ccccc1</smiles>

$\left(\mathbf{2 R , 4 R , 6 S ) - 8 a}\left(\right.\right.$ trans-8a: $\left.\mathbf{R}^{1}=\mathbf{P h}\right)$ : White solid; $\mathrm{R}_{\mathrm{f}}=0.30$ (hexane/EtOAc $\left.=2 / 1\right)$; HPLC analysis Mightysil Si 60

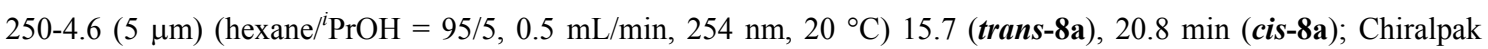
$\mathrm{AD}-\mathrm{H}$ (hexane $\left./ \mathrm{PrOH}=90 / 10,1.0 \mathrm{~mL} / \mathrm{min}, 254 \mathrm{~nm}, 10^{\circ} \mathrm{C}\right) 17.3$ (major), $31.4 \mathrm{~min}(99.5 \%$ ee); Optical rotation; $[\alpha]_{\mathrm{D}}{ }^{26}=-79.6\left(c 1.05, \mathrm{CHCl}_{3}\right) ;{ }^{1} \mathrm{H}$ NMR $\left(\mathrm{CDCl}_{3}, 600 \mathrm{MHz}\right) \delta 1.54(9 \mathrm{H}, \mathrm{s}), 1.63-1.65(2 \mathrm{H}, \mathrm{m}), 2.05(1 \mathrm{H}$, brd, $J=8.4$ Hz), $2.92(1 \mathrm{H}$, brd, $J=13.2 \mathrm{~Hz}), 4.10(1 \mathrm{H}, \mathrm{brs}), 4.63(1 \mathrm{H}, \mathrm{d}, J=6.0 \mathrm{~Hz}), 4.86(1 \mathrm{H}, \mathrm{d}, J=6.0 \mathrm{~Hz}), 4.89-4.96(2 \mathrm{H}, \mathrm{m})$, 5.10-5.16 (2H, m), $5.68\left(1 \mathrm{H}\right.$, brs), $6.25(1 \mathrm{H}, \mathrm{brs}), 7.20-7.47(15 \mathrm{H}, \mathrm{m}) ;\left(\left(\mathrm{CD}_{3}\right)_{2} \mathrm{CO}, 600 \mathrm{MHz}\right) \delta 1.51(9 \mathrm{H}, \mathrm{s}), 1.70(1 \mathrm{H}$, td, $J=12.6,6.0 \mathrm{~Hz}), 1.82(1 \mathrm{H}, \mathrm{td}, J=12.6,5.4 \mathrm{~Hz}), 2.03-2.06(1 \mathrm{H}, \mathrm{m}), 2.91(1 \mathrm{H}, \mathrm{brd}, J=12.6 \mathrm{~Hz}), 4.15-4.22(1 \mathrm{H}$, m), $4.85(2 \mathrm{H}, \mathrm{s}), 5.05-5.12(2 \mathrm{H}, \mathrm{m}), 5.61(1 \mathrm{H}, \mathrm{brd}, J=3.0 \mathrm{~Hz}), 5.71(1 \mathrm{H}, \mathrm{brs}), 6.18(1 \mathrm{H}, \mathrm{brt}, J=5.4 \mathrm{~Hz}), 6.47(1 \mathrm{H}, \mathrm{d}$, $J=6.6 \mathrm{~Hz}), 7.17-7.52(15 \mathrm{H}, \mathrm{m}) ;{ }^{13} \mathrm{C} \mathrm{NMR}\left(\mathrm{CDCl}_{3}, 150.9 \mathrm{MHz}\right) \delta 28.2,33.0,36.7,40.9,50.9,59.3,66.3,66.8,81.1$, 126.3 , 127.2, 127.8, 127.9, 128.1, 128.2, 128.3, 128.5, 128.8, 136.2, 136.3, 140.2, 154.3, 154.7, 155.7; $\left(\left(\mathrm{CD}_{3}\right)_{2} \mathrm{CO}\right.$, $150.9 \mathrm{MHz}) \delta 28.4,33.8,36.5,41.7,51.9,60.3,66.4,66.5,80.7,127.3,127.4,128.5,128.6,128.7,128.8,129.0_{5}$, $129.1_{0}, 129.2,138.1,138.4,142.4,155.2,155.6,156.7$; IR (KBr): 3319, 3063, 2926, 1638, $1535 \mathrm{~cm}^{-1}$; HRMS (ESI) Calcd for $\mathrm{C}_{32} \mathrm{H}_{37} \mathrm{NaN}_{3} \mathrm{O}_{6}\left([\mathrm{M}+\mathrm{Na}]^{+}\right)$582.2575. Found 582.2576.

Configuration Assignment: The absolute stereochemistry was determined to be $6 S$ in comparison with the optical 
rotation, after trans-8a was derivatized from the stereochemically known compound; see Section 2 for details. The relative stereochemistry was assigned by NOE experiments and coupling constants, as shown below.

Selected NOE data and coupling constants of trans-8a $\left(600 \mathrm{MHz},\left(\mathrm{CD}_{3}\right)_{2} \mathrm{CO}\right)$.

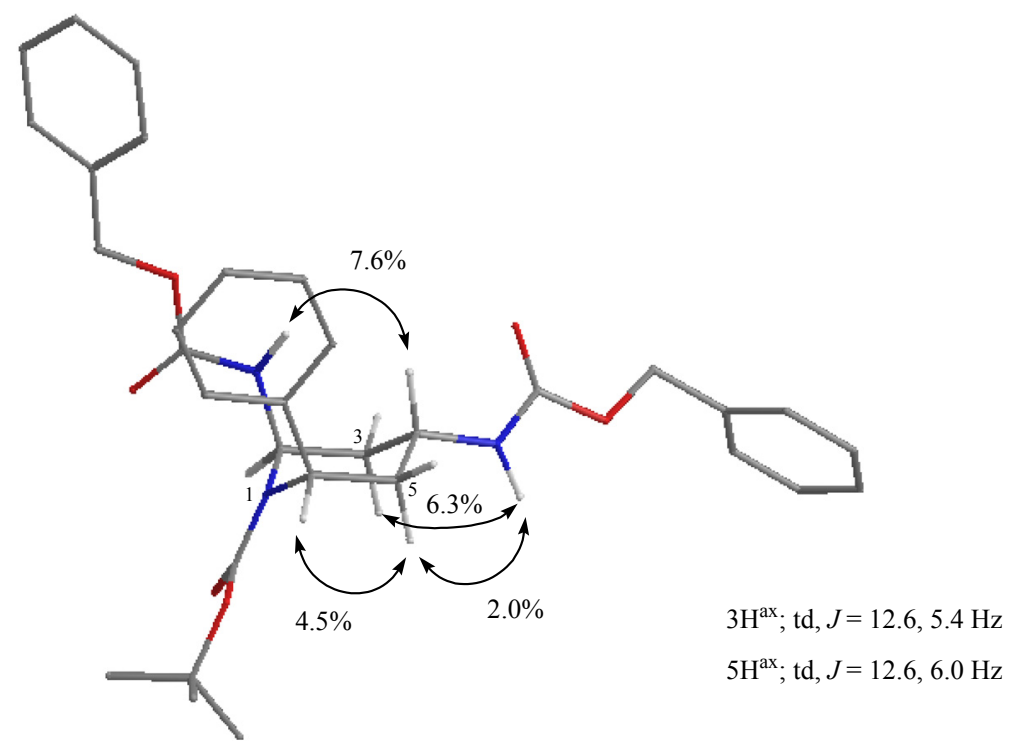

(-Cbz

(2R,4S,6S)-8a (cis-8a: $\left.\mathbf{R}^{1}=\mathbf{P h}\right)$ : White solid; $\mathrm{R}_{\mathrm{f}}=0.25$ (hexane/EtOAc $=2 / 1$ ); HPLC analysis Chiralpak AD-H (hexane $/{ }^{i} \mathrm{PrOH}=90 / 10,1.0 \mathrm{~mL} / \mathrm{min}, 254 \mathrm{~nm}, 10{ }^{\circ} \mathrm{C}$ ) 22.2 (major), $42.4 \mathrm{~min}\left(40.0 \%\right.$ ee); ${ }^{1} \mathrm{H}$ NMR $\left(\mathrm{CDCl}_{3}, 600 \mathrm{MHz}\right)$ $\delta 1.25(9 \mathrm{H}, \mathrm{s}), 1.92(1 \mathrm{H}, \mathrm{brs}), 2.15(1 \mathrm{H}, \mathrm{brs}), 2.27$ (1H, brs), $2.53(1 \mathrm{H}, \mathrm{dt}, J=13.8,7.2 \mathrm{~Hz}), 3.84(1 \mathrm{H}, \mathrm{brs}), 4.94(1 \mathrm{H}$, brs), 5.07-5.14 (5H, m), $5.86\left(1 \mathrm{H}\right.$, brs), $6.05(1 \mathrm{H}, \mathrm{brs}), 7.17-7.22(5 \mathrm{H}, \mathrm{m}), 7.31-7.34(10 \mathrm{H}, \mathrm{m}) ;\left(\left(\mathrm{CD}_{3}\right)_{2} \mathrm{CO}, 600 \mathrm{MHz}\right)$ $\delta 1.28(9 \mathrm{H}, \mathrm{s}), 1.85(1 \mathrm{H}, \mathrm{ddd}, J=14.4,6.6,2.4 \mathrm{~Hz}), 2.20(1 \mathrm{H}, \mathrm{q}, J=13.8 \mathrm{~Hz}), 2.27(1 \mathrm{H}, \mathrm{brs}), 2.67(1 \mathrm{H}, \mathrm{dt}, J=14.4$, $7.8 \mathrm{~Hz}), 3.83(1 \mathrm{H}, \mathrm{brs}), 4.96(1 \mathrm{H}, \mathrm{dd}, J=13.8,6.6 \mathrm{~Hz}), 5.05(2 \mathrm{H}, \mathrm{brs}), 5.11-5.13(2 \mathrm{H}, \mathrm{m}), 6.17(1 \mathrm{H}, \mathrm{td}, J=7.8,2.4$ $\mathrm{Hz}), 6.60(1 \mathrm{H}, \mathrm{brs}), 6.90$ (1H, brs), 7.13-7.36 (15H, m); ${ }^{13} \mathrm{C} \mathrm{NMR}\left(\mathrm{CDCl}_{3}, 150.9 \mathrm{MHz}\right) \delta 28.0,34.9_{2}, 34.9_{2}, 43.8$, $55.7,59.4,66.7_{9}, 66.7_{9}, 80.8,125.3,126.7,127.6,128.1,128.1_{6}, 128.2_{0}, 128.4_{5}, 128.4_{7}, 128.5_{4}, 136.2,136.4,144.0$, $154.8_{4}, 154.8_{4}, 155.7 ;\left(\left(\mathrm{CD}_{3}\right)_{2} \mathrm{CO}, 150.9 \mathrm{MHz}\right) \delta 28.3,35.7,36.1,44.6,57.3,59.8,66.5_{9}, 66.5_{9}, 80.1,126.3_{1}, 126.3_{1}$, 126.9, 128.6 $6_{3}, 128.6_{3}, 128.8_{0}, 128.8_{4}, 129.1_{8}, 129.1_{8}, 138.1_{9}, 138.2_{3}, 146.0,155.2,155.8,156.7$; HRMS (ESI) Calcd for $\mathrm{C}_{32} \mathrm{H}_{37} \mathrm{NaN}_{3} \mathrm{O}_{6}\left([\mathrm{M}+\mathrm{Na}]^{+}\right)$582.2575. Found 582.2576.

Configuration Assignment: The absolute stereochemistry was determined to be $6 S$ in comparison with the retention time of chiral HPLC analysis, after cis-8a was derivatized from the stereochemically known compound; see Section 2 for details. The relative stereochemistry was assigned by NOE experiments and coupling constants, as 
shown below.

Selected NOE data and coupling constants of $\mathbf{c i s}-\mathbf{8 a}\left(600 \mathrm{MHz},\left(\mathrm{CD}_{3}\right)_{2} \mathrm{CO}\right)$

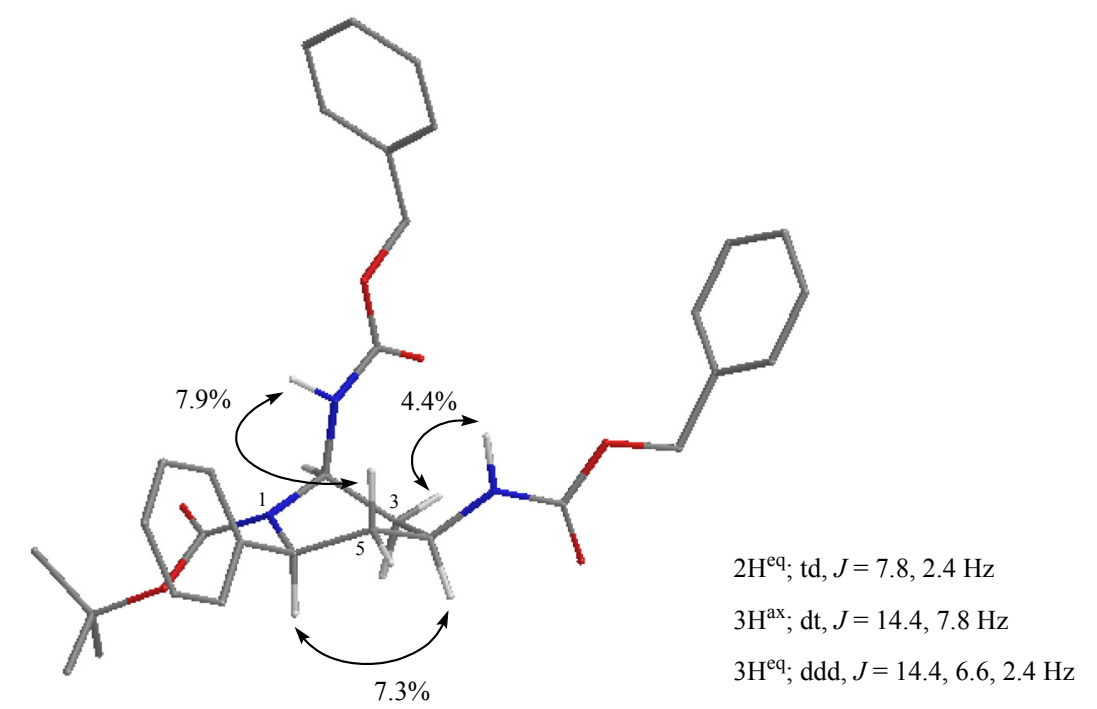

(cbz)

trans-8b $\left(\mathbf{R}^{1}=p\right.$-Br- $\left.\mathbf{C}_{6} \mathbf{H}_{4^{-}}\right)$: The reaction mixture was stirred for $5 \mathrm{~h}$ at $0{ }^{\circ} \mathrm{C}$. White solid; $\mathrm{R}_{\mathrm{f}}=0.35$ (hexane/EtOAc = 2/1); HPLC analysis Mightysil Si 60 250-4.6 (5 $\mu \mathrm{m})\left(\right.$ hexane $/{ }^{i} \mathrm{PrOH}=95 / 5,0.5 \mathrm{~mL} / \mathrm{min}, 254 \mathrm{~nm}$, $\left.20{ }^{\circ} \mathrm{C}\right) 15.9$ (trans-8b), $22.4 \mathrm{~min}\left(\right.$ cis-8b); Chiralcel OD-H (hexane $\left./{ }^{i} \mathrm{PrOH}=90 / 10,1.0 \mathrm{~mL} / \mathrm{min}, 254 \mathrm{~nm}, 20^{\circ} \mathrm{C}\right) 16.5$ (major), $26.6 \mathrm{~min}\left(98.8 \%\right.$ ee); Optical rotation; $[\alpha]_{\mathrm{D}}{ }^{29}=-67.5\left(c \quad 0.98, \mathrm{CHCl}_{3}\right) ;{ }^{1} \mathrm{H} \mathrm{NMR}\left(\mathrm{CDCl}_{3}, 600 \mathrm{MHz}\right) \delta 1.54$ $(9 \mathrm{H}, \mathrm{s}), 1.61-1.66(2 \mathrm{H}, \mathrm{m}), 2.03(1 \mathrm{H}, \mathrm{brd}, J=12.6 \mathrm{~Hz}), 2.86(1 \mathrm{H}, \mathrm{brd}, J=13.2 \mathrm{~Hz}), 4.03(1 \mathrm{H}, \mathrm{brs}), 4.59(1 \mathrm{H}, \mathrm{d}, J=$ $7.2 \mathrm{~Hz}), 4.73(1 \mathrm{H}, \mathrm{d}, J=7.2 \mathrm{~Hz}), 4.88-4.97(2 \mathrm{H}, \mathrm{m}), 5.09-5.16(2 \mathrm{H}, \mathrm{m}), 5.59(1 \mathrm{H}, \mathrm{brs}), 6.23$ (1H, brs), 7.24-7.42 $(14 \mathrm{H}, \mathrm{m}) ;{ }^{13} \mathrm{C} \mathrm{NMR}\left(\mathrm{CDCl}_{3}, 150.9 \mathrm{MHz}\right) \delta 28.2,33.1,36.5,40.9,50.5,59.2,66.6,66.9,81.4,121.2,128.1,128.1_{6}$, $128.1_{6}, 128.3,128.5,128.6,131.8_{6}, 131.8_{6}, 136.0_{9}, 136.0_{9}, 139.5,154.2,154.7,155.7$; IR (KBr): 3346, 3072, 2935 , 1686, 1543, $1072 \mathrm{~cm}^{-1}$; HRMS (ESI) Calcd for $\mathrm{C}_{32} \mathrm{H}_{36} \mathrm{BrNaN}_{3} \mathrm{O}_{6}\left([\mathrm{M}+\mathrm{Na}]^{+}\right)$660.1680, 661.1712. Found 660.1683, 661.1719 .

Configuration Assignment: The absolute stereochemistry was assigned as $6 S$ by analogy. The relative stereochemistry was assigned by NOE experiments and coupling constants. These data were similar to those of trans-8a. 
<smiles>CC(C)(C)OC(=O)NC1CC(NC(=O)OCc2ccccc2)N(C(=O)c2ccccc2)C(c2ccc(Br)cc2)C1</smiles>

cis-8b $\left(\mathbf{R}^{1}=\boldsymbol{p}\right.$-Br- $\left.\mathbf{C}_{6} \mathbf{H}_{4^{-}}\right)$: White solid; $\mathrm{R}_{\mathrm{f}}=0.25$ (hexane/EtOAc $\left.=2 / 1\right)$; HPLC analysis Chiralcel OD-H (hexane $/{ }^{i} \mathrm{PrOH}=90 / 10,1.0 \mathrm{~mL} / \mathrm{min}, 254 \mathrm{~nm}, 20{ }^{\circ} \mathrm{C}$ ) 32.4, 42.5 (major) $\min \left(22.8 \%\right.$ ee); ${ }^{1} \mathrm{H}$ NMR $\left(\mathrm{CDCl}_{3}, 600 \mathrm{MHz}\right)$ $\delta 1.26(9 \mathrm{H}, \mathrm{s}), 1.92(1 \mathrm{H}, \mathrm{brs}), 2.13(1 \mathrm{H}, \mathrm{brs}), 2.20(1 \mathrm{H}, \mathrm{brs}), 2.55(1 \mathrm{H}, \mathrm{dt}, J=12.6,6.0 \mathrm{~Hz}), 3.79(1 \mathrm{H}, \mathrm{brs}), 4.82(1 \mathrm{H}$, brs), 5.07-5.13 (5H, m), $6.04(1 \mathrm{H}, \mathrm{brs}), 7.01(2 \mathrm{H}, \mathrm{brs}), 7.31-7.33(13 \mathrm{H}, \mathrm{m}) ;{ }^{13} \mathrm{C} \mathrm{NMR}\left(\mathrm{CDCl}_{3}, 150.9 \mathrm{MHz}\right) \delta 28.1$, $34.8_{0}, 34.8_{0}, 43.8,55.6,59.2,66.8_{8}, 66.8_{8}, 81.0,120.3,127.0_{1}, 127.0_{3}, 128.2,128.2_{6}, 128.3_{1}, 128.5,128.6,131.4$, 136.2, 136.3, 143.2, 154.6 1 154.6 1 , 155.7; HRMS (ESI) Calcd for $\mathrm{C}_{32} \mathrm{H}_{36} \mathrm{BrNaN}_{3} \mathrm{O}_{6}\left([\mathrm{M}+\mathrm{Na}]^{+}\right) 660.1680,661.1712$. Found 660.1682, 661.1721.

Configuration Assignment: The absolute stereochemistry was assigned as $6 S$ by analogy. The relative stereochemistry was assigned by NOE experiments and coupling constants. These data were similar to those of cis-8a.<smiles>Cc1ccc(C2CC(NC(=O)OC(C)(C)C)CC(NC(=O)OCc3ccccc3)N2C(=O)c2ccccc2)cc1</smiles>

trans-8c $\left(\mathbf{R}^{1}=p\right.$-Me- $\left.\mathbf{C}_{6} \mathbf{H}_{4^{-}}\right)$: The reaction mixture was stirred for $5 \mathrm{~h}$ at $0{ }^{\circ} \mathrm{C}$. White solid; $\mathrm{R}_{\mathrm{f}}=0.30$ (hexane/EtOAc = 2/1); HPLC analysis Mightysil Si 60 250-4.6 $(5 \mu \mathrm{m})\left(\right.$ hexane $/{ }^{i} \mathrm{PrOH}=95 / 5,0.5 \mathrm{~mL} / \mathrm{min}, 254 \mathrm{~nm}$, $\left.20{ }^{\circ} \mathrm{C}\right) 14.8$ (trans-8c), $19.6 \mathrm{~min}\left(\right.$ cis-8c); Chiralcel OD-H (hexane/EtOH = 95/5, $\left.1.0 \mathrm{~mL} / \mathrm{min}, 254 \mathrm{~nm}, 20{ }^{\circ} \mathrm{C}\right) 17.1$ (major), $25.0 \mathrm{~min}\left(98.4 \%\right.$ ee); Optical rotation; $[\alpha]_{\mathrm{D}}{ }^{29}=-83.5\left(c 1.14, \mathrm{CHCl}_{3}\right) ;{ }^{1} \mathrm{H} \mathrm{NMR}\left(\mathrm{CDCl}_{3}, 600 \mathrm{MHz}\right) \delta 1.54$ $(9 \mathrm{H}, \mathrm{s}), 1.60-1.63(2 \mathrm{H}, \mathrm{m}), 2.05(1 \mathrm{H}, \mathrm{brd}, J=12.0 \mathrm{~Hz}), 2.28(3 \mathrm{H}, \mathrm{s}), 2.90(1 \mathrm{H}, \mathrm{brd}, J=13.2 \mathrm{~Hz}), 4.09$ (1H, brs), 4.64 $(1 \mathrm{H}, \mathrm{d}, J=7.8 \mathrm{~Hz}), 4.82(1 \mathrm{H}, \mathrm{d}, J=6.6 \mathrm{~Hz}), 4.88-4.99$ (2H, m), 5.10-5.16 (2H, m), 5.64 (1H, brs), 6.25 (1H, brs), $7.12(2 \mathrm{H}, \mathrm{d}, J=7.2 \mathrm{~Hz}), 7.21(2 \mathrm{H}, \mathrm{d}, J=7.2 \mathrm{~Hz}), 7.29-7.41(10 \mathrm{H}, \mathrm{m}) ;{ }^{13} \mathrm{C} \mathrm{NMR}\left(\mathrm{CDCl}_{3}, 150.9 \mathrm{MHz}\right) \delta 20.9,28.2$, $33.2,36.8,40.9,50.8,59.3,66.3,66.8,81.1,126.2,127.7,127.9,128.1,128.2,128.3,128.6,129.5,136.2,136.3$, 136.8, 137.1, 154.3, 154.7, 155.7; IR (KBr): 3329, 3032, 2991, 1686, $1547 \mathrm{~cm}^{-1}$; HRMS (ESI) Calcd for $\mathrm{C}_{33} \mathrm{H}_{39} \mathrm{NaN}_{3} \mathrm{O}_{6}\left([\mathrm{M}+\mathrm{Na}]^{+}\right)$596.2731. Found 596.2731.

Configuration Assignment: The absolute stereochemistry was assigned as $6 S$ by analogy. The relative stereochemistry was assigned by NOE experiments and coupling constants. These data were similar to those of trans-8a.<smiles>Cc1ccc(C2CC(NC(=O)OC(C)(C)C)CC(NC(=O)OCc3ccccc3)N2C(=O)c2ccccc2)cc1</smiles> 
cis-8c $\left(\mathbf{R}^{1}=\boldsymbol{p}\right.$-Me- $\left.\mathbf{C}_{6} \mathbf{H}_{4^{-}}\right)$: White solid; $\mathrm{R}_{\mathrm{f}}=0.25$ (hexane/EtOAc $\left.=2 / 1\right)$; HPLC analysis Chiralcel OD-H (hexane/EtOH = 95/5, $1.0 \mathrm{~mL} / \mathrm{min}, 254 \mathrm{~nm}, 20{ }^{\circ} \mathrm{C}$ ) 39.0 (major), $55.0 \mathrm{~min}\left(4.3 \%\right.$ ee); ${ }^{1} \mathrm{H} \mathrm{NMR}\left(\mathrm{CDCl}_{3}, 600 \mathrm{MHz}\right) \delta$ 1.29 (9H, s), 1.89 (1H, brs), 2.14 (1H, brd, $J=10.8 \mathrm{~Hz}), 2.30$ (4H, brs), 2.49 (1H, dt, $J=14.4,7.2 \mathrm{~Hz}), 3.83(1 \mathrm{H}$, brs), $4.99(1 \mathrm{H}, \mathrm{brs}), 5.06-5.14(5 \mathrm{H}, \mathrm{m}), 5.71$ (1H, brs), 6.04 (1H, brs), 7.03-7.06 (4H, m), 7.31-7.34 (10H, m); ${ }^{13} \mathrm{C} \mathrm{NMR}$ $\left(\mathrm{CDCl}_{3}, 150.9 \mathrm{MHz}\right) \delta 20.9,28.1,34.6,35.1,43.9,54.9,59.5,66.7_{8}, 66.7_{8}, 80.8,125.3,128.1,128.1_{5}, 128.1_{8}, 128.2_{0}$, $128.4_{7}, 128.5_{4}, 129.2,136.3,136.4,140.6_{8}, 140.6_{8}, 154.8_{9}, 154.8_{9}, 155.6$; HRMS (ESI) Calcd for $\mathrm{C}_{33} \mathrm{H}_{39} \mathrm{NaN}_{3} \mathrm{O}_{6}$ ([M $+\mathrm{Na}]^{+}$) 596.2731. Found 596.2733.

Configuration Assignment: The absolute stereochemistry was assigned as $6 S$ by analogy. The relative stereochemistry was assigned by NOE experiments and coupling constants. These data were similar to those of cis-8a.

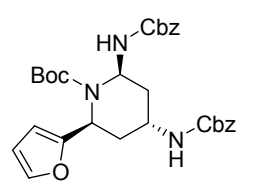

trans-8d ( $\mathbf{R}^{1}=$ 2-furyl): Reaction run using $5 \mathrm{~mol} \%$ of $(R)-3 \mathbf{d}(3.26 \mathrm{mg}, 0.005 \mathrm{mmol})$. The reaction mixture was stirred for $5 \mathrm{~h}$ at $0{ }^{\circ} \mathrm{C}$. White solid; $\mathrm{R}_{\mathrm{f}}=0.25$ (hexane/EtOAc $\left.=2 / 1\right)$; HPLC analysis Mightysil Si $60250-4.6(5 \mu \mathrm{m})$ (hexane $/{ }^{i} \mathrm{PrOH}=97 / 3,0.5 \mathrm{~mL} / \mathrm{min}, 254 \mathrm{~nm}, 20^{\circ} \mathrm{C}$ ) 32.1 (trans-8d), $36.2 \mathrm{~min}$ (cis-8d); Chiralpak IA (hexane $/{ }^{i} \mathrm{PrOH}$ $\left.=85 / 15,1.0 \mathrm{~mL} / \mathrm{min}, 254 \mathrm{~nm}, 20{ }^{\circ} \mathrm{C}\right) 11.4$ (major), $22.6 \mathrm{~min}\left(98.6 \%\right.$ ee); Optical rotation; $[\alpha]_{\mathrm{D}}{ }^{30}=-50.8(c 0.86$, $\left.\mathrm{CHCl}_{3}\right) ;{ }^{1} \mathrm{H} \mathrm{NMR}\left(\mathrm{CDCl}_{3}, 600 \mathrm{MHz}\right) \delta 1.46(9 \mathrm{H}, \mathrm{s}), 1.59-1.68(2 \mathrm{H}, \mathrm{m}), 2.15(1 \mathrm{H}, \mathrm{brs}), 2.54(1 \mathrm{H}, \mathrm{brd}, J=12.6 \mathrm{~Hz})$, 4.27 (1H, brs), $4.67(1 \mathrm{H}, \mathrm{d}, J=7.2 \mathrm{~Hz}), 4.98(1 \mathrm{H}, \mathrm{d}, J=12.0 \mathrm{~Hz}), 5.08-5.13(4 \mathrm{H}, \mathrm{m}), 5.50$ (1H, brs), $6.12(1 \mathrm{H}, \mathrm{brs})$, $6.33(1 \mathrm{H}, \mathrm{brs}), 6.39$ (1H, brs), 7.28-7.38 (11H, m); ${ }^{13} \mathrm{C} \mathrm{NMR}\left(\mathrm{CDCl}_{3}, 150.9 \mathrm{MHz}\right) \delta 28.2,33.9,37.1,41.3,47.0,59.2$, 66.4, 66.8, 81.3, 107.6, 110.8, 127.9 $, 127.9_{3}, 128.2,128.3,128.4,128.6,136.2,136.5,142.2,153.8,154.2,154.7$, 155.6; IR (KBr): 3346, 3032, 2966, 1686, $1526 \mathrm{~cm}^{-1}$; HRMS (ESI) Calcd for $\mathrm{C}_{30} \mathrm{H}_{35} \mathrm{NaN}_{3} \mathrm{O}_{7}\left([\mathrm{M}+\mathrm{Na}]^{+}\right)$572.2367. Found 572.2369.

Configuration Assignment: The absolute stereochemistry was assigned as $6 S$ by analogy. The relative stereochemistry was assigned by NOE experiments and coupling constants. These data were similar to those of trans-8a.

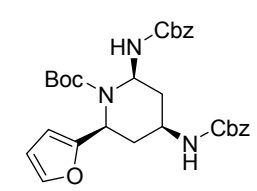

cis-8d $\left(\mathbf{R}^{1}=\right.$ 2-furyl): White solid; $\mathrm{R}_{\mathrm{f}}=0.25$ (hexane/EtOAc $\left.=2 / 1\right)$; HPLC analysis Chiralpak IA (hexane $/{ }^{i} \operatorname{PrOH}=$ 85/15, $\left.1.0 \mathrm{~mL} / \mathrm{min}, 254 \mathrm{~nm}, 20{ }^{\circ} \mathrm{C}\right) 14.8,18.8$ (major) $\min \left(13.7 \%\right.$ ee); ${ }^{1} \mathrm{H} \mathrm{NMR}\left(\mathrm{CDCl}_{3}, 600 \mathrm{MHz}\right) \delta 1.41(9 \mathrm{H}, \mathrm{s})$, $1.97(1 \mathrm{H}, \mathrm{brs}), 2.19-2.45(2 \mathrm{H}, \mathrm{m}), 2.29-2.34(1 \mathrm{H}, \mathrm{m}), 3.88$ (1H, brd, $J=4.8 \mathrm{~Hz}), 4.98$ (1H, brs), 5.03-5.13 (4H, m), $5.33(1 \mathrm{H}, \mathrm{brs}), 5.40(1 \mathrm{H}, \mathrm{dd}, J=7.8,4.2 \mathrm{~Hz}), 5.92(1 \mathrm{H}, \mathrm{td}, J=10.8,6.0 \mathrm{~Hz}), 6.14(1 \mathrm{H}, \mathrm{brd}, J=4.2 \mathrm{~Hz}), 6.30(1 \mathrm{H}$, brs), 7.29-7.36 (11H, m); ${ }^{13} \mathrm{C} \mathrm{NMR}\left(\mathrm{CDCl}_{3}, 150.9 \mathrm{MHz}\right) \delta 28.2,31.6,34.6,42.9,46.3,58.8,66.6_{9}, 66.6_{9}, 81.4,106.8$, 
$111.1,128.0_{9}, 128.1_{2}, 128.1_{5}, 128.1_{7}, 128.4_{7}, 128.5_{2}, 136.3,136.4,141.5,154.4,154.6,154.8,155.5$; HRMS (ESI) Calcd for $\mathrm{C}_{30} \mathrm{H}_{35} \mathrm{NaN}_{3} \mathrm{O}_{7}\left([\mathrm{M}+\mathrm{Na}]^{+}\right)$572.2367. Found 572.2369.

Configuration Assignment: The absolute stereochemistry was assigned as $6 S$ by analogy. The relative stereochemistry was assigned by NOE experiments and coupling constants. These data were similar to those of cis-8a.

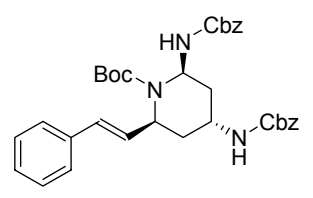

trans-8e ( $\left.\mathbf{R}^{\mathbf{1}}=\mathbf{P h}-\mathbf{C H}=\mathbf{C H}-\right)$ : Reaction run using $5 \mathrm{~mol} \%$ of $(R)-\mathbf{3 d}(3.26 \mathrm{mg}, 0.005 \mathrm{mmol})$. The reaction mixture was stirred for $5 \mathrm{~h}$ at $0{ }^{\circ} \mathrm{C}$. White solid; $\mathrm{R}_{\mathrm{f}}=0.30$ (hexane/EtOAc $\left.=2 / 1\right)$; HPLC analysis Mightysil Si 60 250-4.6 (5 $\mu \mathrm{m})\left(\right.$ hexane $\left./{ }^{i} \mathrm{PrOH}=95 / 5,0.5 \mathrm{~mL} / \mathrm{min}, 254 \mathrm{~nm}, 20^{\circ} \mathrm{C}\right) 14.6$ (trans-8e), $24.4 \mathrm{~min}$ (cis-8e); Chiralcel OD-H (hexane/EtOH $=90 / 10,1.0 \mathrm{~mL} / \mathrm{min}, 254 \mathrm{~nm}, 30{ }^{\circ} \mathrm{C}$ ) 10.6 (major), $12.4 \mathrm{~min}\left(96.8 \%\right.$ ee); Optical rotation; $[\alpha]_{\mathrm{D}}{ }^{28}=$ -41.5 (c 1.01, $\left.\mathrm{CHCl}_{3}\right) ;{ }^{1} \mathrm{H} \mathrm{NMR}\left(\mathrm{CDCl}_{3}, 600 \mathrm{MHz}\right) \delta 1.48(9 \mathrm{H}, \mathrm{s}), 1.57-1.62(2 \mathrm{H}, \mathrm{m}), 2.16(1 \mathrm{H}, \mathrm{brd}, J=13.8 \mathrm{~Hz})$, $2.46(1 \mathrm{H}$, brd, $J=12.6 \mathrm{~Hz}), 4.10(1 \mathrm{H}, \mathrm{brs}), 4.67(1 \mathrm{H}, \mathrm{d}, J=7.2 \mathrm{~Hz}), 5.00(1 \mathrm{H}, \mathrm{brd}, J=12.0 \mathrm{~Hz}), 5.10-5.14(4 \mathrm{H}, \mathrm{m})$, $5.31(1 \mathrm{H}, \mathrm{brs}), 6.15(1 \mathrm{H}, \mathrm{brs}), 6.26(1 \mathrm{H}, \mathrm{dd}, J=16.2,4.2 \mathrm{~Hz}), 6.70(1 \mathrm{H}, \mathrm{d}, J=16.2 \mathrm{~Hz}), 7.23-7.38(15 \mathrm{H}, \mathrm{m}) ;{ }^{13} \mathrm{C}$ $\operatorname{NMR}\left(\mathrm{CDCl}_{3}, 150.9 \mathrm{MHz}\right) \delta 28.2,34.6,37.1,40.8,50.9,59.7,66.7,66.9,81.2,126.6,127.8_{7}, 127.8_{7}, 128.0_{0}, 128.0_{0}$, 128.2, 128.3, 128.4, 128.5 , 128.5 , 129.3, 131.7, 136.2, 136.3, 154.2, 154.5, 155.6; IR (KBr): 3327, 3032, 2974, 1701, 1543, $1028 \mathrm{~cm}^{-1}$; HRMS (ESI) Calcd for $\mathrm{C}_{34} \mathrm{H}_{39} \mathrm{NaN}_{3} \mathrm{O}_{6}\left([\mathrm{M}+\mathrm{Na}]^{+}\right)$608.2731. Found 608.2732.

Configuration Assignment: The absolute stereochemistry was assigned as $6 S$ by analogy. The relative stereochemistry was assigned by NOE experiments and coupling constants. These data were similar to those of trans-8a.<smiles></smiles>

cis-8e $\left(\mathbf{R}^{1}=\mathbf{P h}-\mathbf{C H}=\mathbf{C H}-\right)$ : White solid; $\mathrm{R}_{\mathrm{f}}=0.25$ (hexane/EtOAc $\left.=2 / 1\right) ;$ HPLC analysis Chiralcel OD-H (hexane/EtOH $\left.=90 / 10,1.0 \mathrm{~mL} / \mathrm{min}, 254 \mathrm{~nm}, 30{ }^{\circ} \mathrm{C}\right) 19.2$ (major), $45.2 \min \left(35.7 \%\right.$ ee); ${ }^{1} \mathrm{H} \mathrm{NMR}\left(\mathrm{CDCl}_{3}, 600 \mathrm{MHz}\right)$ $\delta 1.44(9 \mathrm{H}, \mathrm{s}), 1.96(1 \mathrm{H}, \mathrm{brs}), 2.06(1 \mathrm{H}, \mathrm{brs}), 2.16-2.20(1 \mathrm{H}, \mathrm{m}), 2.33(1 \mathrm{H}, \mathrm{dt}, J=13.2,6.6 \mathrm{~Hz}), 3.84(1 \mathrm{H}, \mathrm{dd}, J=$ 12.0, 6.6 Hz), $4.82(1 \mathrm{H}, \mathrm{dd}, J=12.0,5.4 \mathrm{~Hz}), 5.05-5.12(5 \mathrm{H}, \mathrm{m}), 5.41(1 \mathrm{H}, \mathrm{brs}), 5.92(1 \mathrm{H}, \mathrm{td}, J=6.6,3.6 \mathrm{~Hz}), 6.22$ $(1 \mathrm{H}, \mathrm{dd}, J=15.6,5.4 \mathrm{~Hz}), 6.46(1 \mathrm{H}, \mathrm{dd}, J=15.6,1.2 \mathrm{~Hz}), 7.21-7.35(15 \mathrm{H}, \mathrm{m}) ;{ }^{13} \mathrm{C} \mathrm{NMR}\left(\mathrm{CDCl}_{3}, 150.9 \mathrm{MHz}\right) \delta 28.3$, 33.0, 34.6, 43.7, 51.6, 59.2, 66.8 $, 66.8_{0}, 81.0,126.4,127.7,128.1_{2}, 128.1_{2}, 128.1_{6}, 128.2_{0}, 128.4_{8}, 128.4_{8}, 128.5_{4}$, 128.6, 130.6, 131.3, 136.2, 136.4, 154.6, 155.0, 155.6; HRMS (ESI) Calcd for $\mathrm{C}_{34} \mathrm{H}_{39} \mathrm{NaN}_{3} \mathrm{O}_{6}\left([\mathrm{M}+\mathrm{Na}]^{+}\right) 608.2731$. Found 608.2729.

Configuration Assignment: The absolute stereochemistry was assigned as $6 S$ by analogy. The relative stereochemistry was assigned by NOE experiments and coupling constants. These data were similar to those of 
cis-8a.

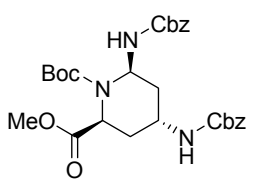

trans-8f ( $\left.\mathbf{R}^{1}=\mathbf{M e O}_{2} \mathbf{C}-\right)$ : Reaction run using $5 \mathrm{~mol} \%$ of $(R)-\mathbf{3 d}(3.26 \mathrm{mg}, 0.005 \mathrm{mmol})$. The reaction mixture was stirred for $5 \mathrm{~h}$ at $0{ }^{\circ} \mathrm{C}$. White solid; $\mathrm{R}_{\mathrm{f}}=0.20$ (hexane/EtOAc $\left.=2 / 1\right)$; HPLC analysis Inertsil SIL 100A $(3 \mu \mathrm{m})$ (hexane $/{ }^{i} \mathrm{PrOH}=95 / 5,0.7 \mathrm{~mL} / \mathrm{min}, 254 \mathrm{~nm}, 30{ }^{\circ} \mathrm{C}$ ) 16.4 (trans-8f), $17.8 \mathrm{~min}$ (cis-8f); Chiralcel OD-H (hexane $/{ }^{i} \mathrm{PrOH}=80 / 20,1.0 \mathrm{~mL} / \mathrm{min}, 254 \mathrm{~nm}, 20{ }^{\circ} \mathrm{C}$ ) 9.4 (major), $39.3 \mathrm{~min}\left(98.2 \%\right.$ ee); Optical rotation; $[\alpha]_{\mathrm{D}}{ }^{31}=$ -10.7 (c 1.13, $\left.\mathrm{CHCl}_{3}\right) ;{ }^{1} \mathrm{H}$ NMR $\left(\mathrm{CDCl}_{3}, 600 \mathrm{MHz}\right) \delta 1.36(9 \mathrm{H}, \mathrm{s}), 1.55-1.62(2 \mathrm{H}, \mathrm{m}), 2.17(1 \mathrm{H}$, brd, $J=7.8 \mathrm{~Hz})$, $2.47(1 \mathrm{H}$, brd, $J=12.6 \mathrm{~Hz}), 3.79$ (4H, brs), $4.71(1 \mathrm{H}, \mathrm{brs}), 4.84(1 \mathrm{H}, \mathrm{brs}), 5.02-5.18(4 \mathrm{H}, \mathrm{m}), 5.86(1 \mathrm{H}, \mathrm{brs}), 7.07(1 \mathrm{H}$, brs), 7.28-7.39 (10H, m); ${ }^{13} \mathrm{C} \mathrm{NMR}\left(\mathrm{CDCl}_{3}, 150.9 \mathrm{MHz}\right) \delta 28.0,32.9,37.2,41.2,53.0,54.4,60.1,66.4,66.8,81.9$, 127.9, 128.1, 128.1 1 , 128.2 1 , 128.4, 128.6, 136.2, 136.7, 154.9, 155.1, 155.4, 174.9; IR (KBr): 3368, 3032, 2966, 1726, 1701, 1543, $1213 \mathrm{~cm}^{-1}$; HRMS (ESI) Calcd for $\mathrm{C}_{28} \mathrm{H}_{35} \mathrm{NaN}_{3} \mathrm{O}_{8}\left([\mathrm{M}+\mathrm{Na}]^{+}\right)$564.2316. Found 564.2318.

Configuration Assignment: The absolute stereochemistry was assigned as $6 S$ by analogy. The relative stereochemistry was assigned by NOE experiments and coupling constants. These data were similar to those of trans-8a.<smiles>O=C(NC1CC(NC(=O)c2ccccc2)C(NC(=O)c2ccccc2)CC1C1CCCCC1)OCc1ccccc1</smiles>

trans-8g ( $\left.\mathbf{R}^{\mathbf{1}}=\boldsymbol{c}-\mathbf{C}_{\mathbf{6}} \mathbf{H}_{11}\right)$ : Reaction run using $5 \mathrm{~mol} \%$ of $(R)-\mathbf{3 d}(3.26 \mathrm{mg}, 0.005 \mathrm{mmol})$. The reaction mixture was stirred for $5 \mathrm{~h}$ at $0{ }^{\circ} \mathrm{C}$. White solid; $\mathrm{R}_{\mathrm{f}}=0.35$ (hexane/EtOAc $\left.=2 / 1\right)$; HPLC analysis Mightysil Si 60 250-4.6 (5 $\left.\mu \mathrm{m}\right)$ (hexane/EtOAc $=80 / 20,0.7 \mathrm{~mL} / \mathrm{min}, 254 \mathrm{~nm}, 20{ }^{\circ} \mathrm{C}$ ) 22.0 (trans-8g), $27.9 \min$ (cis-8g); Chiralcel OD-H (hexane $\left./{ }^{i} \mathrm{PrOH}=90 / 10,0.5 \mathrm{~mL} / \mathrm{min}, 254 \mathrm{~nm}, 20^{\circ} \mathrm{C}\right) 13.9$ (major), $19.4 \mathrm{~min}\left(97.0 \%\right.$ ee); Optical rotation; $[\alpha]_{\mathrm{D}}{ }^{31}=$ -9.3 (c 0.98, $\left.\mathrm{CHCl}_{3}\right)$; ${ }^{1} \mathrm{H}$ NMR $\left(\mathrm{CDCl}_{3}, 600 \mathrm{MHz}\right) \delta 0.88(1 \mathrm{H}, \mathrm{brs}), 1.05-1.12(3 \mathrm{H}, \mathrm{m}), 1.25-1.27(2 \mathrm{H}, \mathrm{m}), 1.45(10 \mathrm{H}$, brs), 1.48-1.53 (1H, m), 1.62-1.67 (3H, m), $1.75(1 \mathrm{H}, \mathrm{d}, J=12.0 \mathrm{~Hz}), 1.88(1 \mathrm{H}, \mathrm{d}, J=10.8 \mathrm{~Hz}), 2.14(1 \mathrm{H}, \mathrm{brd}, J=$ $9.6 \mathrm{~Hz}), 2.25$ (1H, brd, $J=12.6 \mathrm{~Hz}), 3.91(1 \mathrm{H}, \mathrm{brs}), 4.08$ (1H, brs), 4.57 (1H, d, $J=6.6 \mathrm{~Hz}), 5.06-5.17(5 \mathrm{H}, \mathrm{m}), 6.20$ (1H, brs), 7.31-7.37 (10H, m); ${ }^{13} \mathrm{C} \mathrm{NMR}\left(\mathrm{CDCl}_{3}, 150.9 \mathrm{MHz}\right) \delta 26.0_{6}, 26.0_{8}, 26.2,28.2,29.7,31.3,32.4,37.5,40.8$, 41.0, 55.1, 59.0, 66.8 $3,66.8_{3}, 80.5,128.1,128.2,128.3,128.4,128.5,128.6,136.3,136.4,154.7,154.8,155.5$; IR (KBr): 3329, 3040, 2930, 2854, 1697, $1529 \mathrm{~cm}^{-1}$; HRMS (ESI) Calcd for $\mathrm{C}_{32} \mathrm{H}_{43} \mathrm{NaN}_{3} \mathrm{O}_{6}\left([\mathrm{M}+\mathrm{Na}]^{+}\right) 588.3044$. Found 588.3042 .

Configuration Assignment: The absolute stereochemistry was assigned as $6 S$ by analogy. The relative stereochemistry was assigned by NOE experiments and coupling constants. These data were similar to those of trans-8a. 


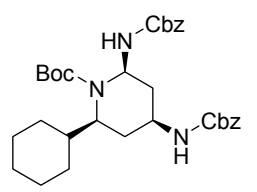

cis-8g $\left(\mathbf{R}^{1}=c-\mathbf{C}_{\mathbf{6}} \mathbf{H}_{11}\right.$ )): White solid; $\mathrm{R}_{\mathrm{f}}=0.35$ (hexane/EtOAc $\left.=2 / 1\right)$; HPLC analysis Chiralcel OD-H (hexane $/{ }^{i} \operatorname{PrOH}$ $\left.=90 / 10,0.5 \mathrm{~mL} / \mathrm{min}, 254 \mathrm{~nm}, 20{ }^{\circ} \mathrm{C}\right) 31.4$ (major), $55.1 \mathrm{~min}\left(48.3 \%\right.$ ee); ${ }^{1} \mathrm{H}$ NMR $\left(\mathrm{CDCl}_{3}, 600 \mathrm{MHz}\right) \delta 0.86-0.89$ $(1 \mathrm{H}, \mathrm{m}), 0.99-1.26(5 \mathrm{H}, \mathrm{m}), 1.43-1.51(11 \mathrm{H}, \mathrm{m}), 1.60-1.71(5 \mathrm{H}, \mathrm{m}), 2.13-2.17(1 \mathrm{H}, \mathrm{m}), 2.41(1 \mathrm{H}, \mathrm{dt}, J=13.8,6.6 \mathrm{~Hz})$, 3.58-3.65 (1H, m), 3.86-3.89 (1H, m), 4.80 (1H, brs), 5.09 (2H, brs), $5.10(2 \mathrm{H}, \mathrm{brs}), 5.26(1 \mathrm{H}, \mathrm{brs}), 5.86(1 \mathrm{H}, \mathrm{brs})$, 7.29-7.37 (10H, m); ${ }^{13} \mathrm{C} \mathrm{NMR}\left(\mathrm{CDCl}_{3}, 150.9 \mathrm{MHz}\right) \delta 26.1,26.2,26.3,28.3,30.0,30.2,31.6,35.4,44.5,45.0,55.5$, $60.1,66.7,66.8,80.6,128.1_{6}, 128.1_{8}, 128.2,128.5,128.5_{6}, 128.6_{0}, 136.3,136.5,154.9,155.3,155.6$; HRMS (ESI) Calcd for $\mathrm{C}_{32} \mathrm{H}_{43} \mathrm{NaN}_{3} \mathrm{O}_{6}\left([\mathrm{M}+\mathrm{Na}]^{+}\right)$588.3044. Found 588.3045.

Configuration Assignment: The absolute stereochemistry was assigned as $6 S$ by analogy. The relative stereochemistry was assigned by NOE experiments and coupling constants. These data were similar to those of cis-8a.

\section{Determination of Absolute Configuration of Piperidine Derivatives $\left(8 \mathrm{~B} ; \mathbf{R}^{\mathbf{1}}=\mathbf{P h}\right)$ :}

The absolute stereochemistry was determined through chemical transformations in which an optically active aminal (10) was derivatized to either the stereochemically known compound (12) or piperidine derivatives (8a) as shown in the following scheme. To determine the absolute configuration of the optically active aminal (10), 10 was transformed to the stereochemically known amino alcohol (12) by two step operations via aldehyde (11). The optically active aminal (10), thus determined the absolute stereochemistry, was reacted with mono-substituted enecarbamate (5a) in the presence of biphenol-derived phosphoric acid (9), giving the corresponding piperidine derivatives (8a) as the stereochemically known form.

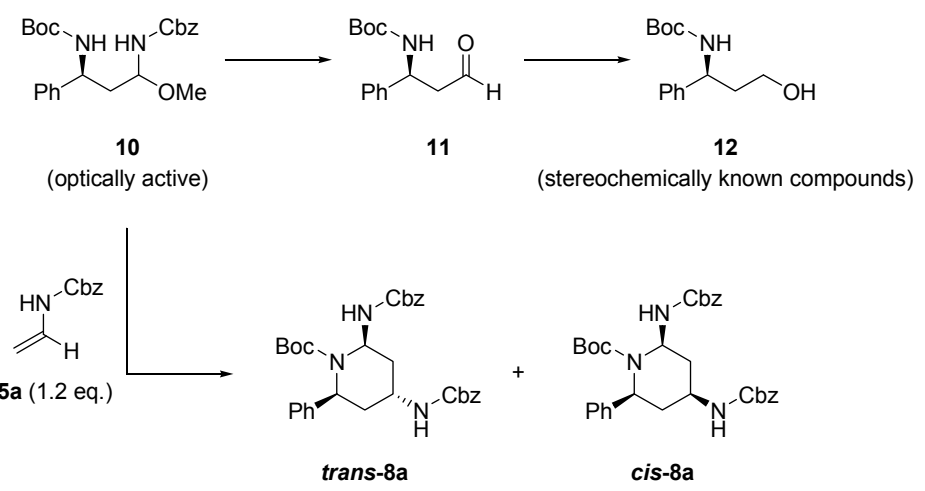

2-1 Transformation of Optically Active Aminal (10) to Stereochemically Known Amino Alcohol (12).

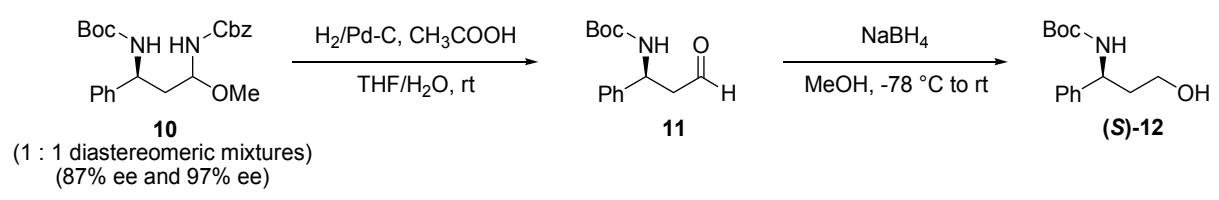


Reduction of 10: To a dried two neck flask was weighted 10 (216.5 mg, $0.52 \mathrm{mmol}, 1: 1$ diastereomeric mixtures, $87 \%$ ee and $97 \%$ ee). The atmosphere was replaced by nitrogen. After $\mathbf{1 0}$ was dissolved in tetrahydrofuran/water $(2 / 1,3.0 \mathrm{~mL})$, acetic acid $(297.7 \mu \mathrm{L}, 5.2 \mathrm{mmol})$ and Pd-C 5w\% $(110.7 \mathrm{mg}, 0.052 \mathrm{mmol})$ were introduced at $0{ }^{\circ} \mathrm{C}$. Then the nitrogen was replaced by hydrogen. The reaction mixture was stirred for $24 \mathrm{~h}$ at room temperature. The reaction was filtrated. After being concentrated, the resulting residure was used for the next reaction without further purification.

To a methanol $(5.0 \mathrm{~mL})$ solution of the crude 11 was added $\mathrm{NaBH}_{4}$ at $-78{ }^{\circ} \mathrm{C}$. After stirring for $12 \mathrm{~h}$ at room temperature, the reaction mixture was quenched by addition of saturated aqueous $\mathrm{NaHCO}_{3}$. The resulting mixture was extracted with dichloromethane, dried over anhydrous $\mathrm{Na}_{2} \mathrm{SO}_{4}$, and evaporated under reduced pressure. Purification of the residue using chromatography (dichloromethane/methanol =100/1-50/1 as eluent) gave 12 as white solid in $5 \%$ yield $(90.0 \%$ ee).

12: White solid; $\mathrm{R}_{\mathrm{f}}=0.75$ (dichloromethane/methanol $=10 / 1$ ); HPLC analysis Chiralpak AD-H (hexane $/{ }^{i} \mathrm{PrOH}=$ 90/10, $\left.1.0 \mathrm{~mL} / \mathrm{min}, 254 \mathrm{~nm}, 10^{\circ} \mathrm{C}\right) 11.7$ (major), $19.5 \mathrm{~min} ;{ }^{1} \mathrm{H}$ NMR $\left(\mathrm{CDCl}_{3}, 270 \mathrm{MHz}\right) \delta 1.42(9 \mathrm{H}, \mathrm{s}), 1.77-1.86$ $(1 \mathrm{H}, \mathrm{m}), 2.02-2.14(1 \mathrm{H}, \mathrm{m}), 3.14(1 \mathrm{H}, \mathrm{brs}), 3.69-3.72(2 \mathrm{H}, \mathrm{m}), 4.89-4.97(2 \mathrm{H}, \mathrm{m}), 7.27-7.38(5 \mathrm{H}, \mathrm{m}) ;{ }^{13} \mathrm{C} \mathrm{NMR}$ $\left(\mathrm{CDCl}_{3}, 67.8 \mathrm{MHz}\right) \delta 28.4,39.5,51.5,59.0,80.0,126.1,126.3,127.4,128.7,141.8$; HRMS (ESI) Calcd for $\mathrm{C}_{14} \mathrm{H}_{21} \mathrm{NaNO}_{3}\left([\mathrm{M}+\mathrm{Na}]^{+}\right)$274.1414. Found 274.1412.

Absolute configuration was determined to be $(S)$ by optical rotation; $[\alpha]_{\mathrm{D}}^{23}=-30.9\left(\mathrm{c} 0.7, \mathrm{CHCl}_{3}\right)\left\{\right.$ lit $^{6}$., $(R)-\mathbf{1 2},[\alpha]_{\mathrm{D}}{ }^{25}$ $\left.=+55.4\left(\mathrm{c} 1.0, \mathrm{CHCl}_{3}\right)\right\}$.

\section{2-2 Transformation of Optically Active Aminal (10) to 8a $\left(\mathbf{R}^{1}=\mathbf{P h}\right)$}

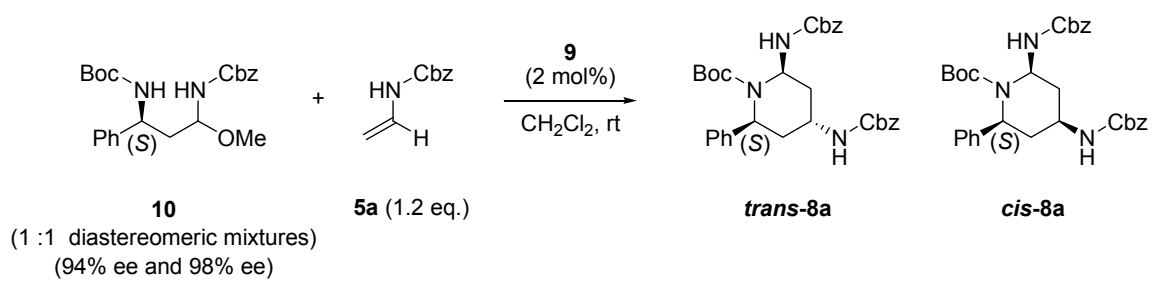

To a dried test tube was weighted chiral phosphoric acid $\mathbf{9}(1.35 \mathrm{mg}, 0.0054 \mathrm{mmol})$ and $\mathbf{1 0}(114.6 \mathrm{mg}, 0.27 \mathrm{mmol}, 1$ : 1 diastereomeric mixtures, $94 \%$ ee and $98 \%$ ee). The atmosphere was replaced by nitrogen. After catalyst 9 and $\mathbf{1 0}$ were dissolved in dichloromethane $(2.5 \mathrm{~mL})$, enecarbamate $\mathbf{5 a}(52.6 \mathrm{mg}, 0.30 \mathrm{mmol})$ was introduced at room temperature. The reaction mixture was stirred for $2 \mathrm{~h}$ at room temperature. The reaction was quenched by addition of saturated aqueous $\mathrm{NaHCO}_{3}$, and then the resulting mixture was extracted with dichloromethane and dried over anhydrous $\mathrm{Na}_{2} \mathrm{SO}_{4}$. After the solvents were evaporated, the crude material was purified by chromatography (hexane/EtOAc $=8 / 1-1 / 1$ as eluent) to give piperidine derivatives $8 \mathbf{a}\left(\mathrm{R}^{1}=\mathrm{Ph}\right)$ in $49 \%$ yield $($ trans-8a/cis-8a $=80$ $(91 \%$ ee $) / 20(95 \%$ ee $))$ as a white solid.

(6) Stephen, G. D.; A. Christopher, G.; Paul, M. R.; Andrew, D. S.;Miles, J. S.; James, E. T. Org. Biomol. Chem. 2006, 4, 2753. 


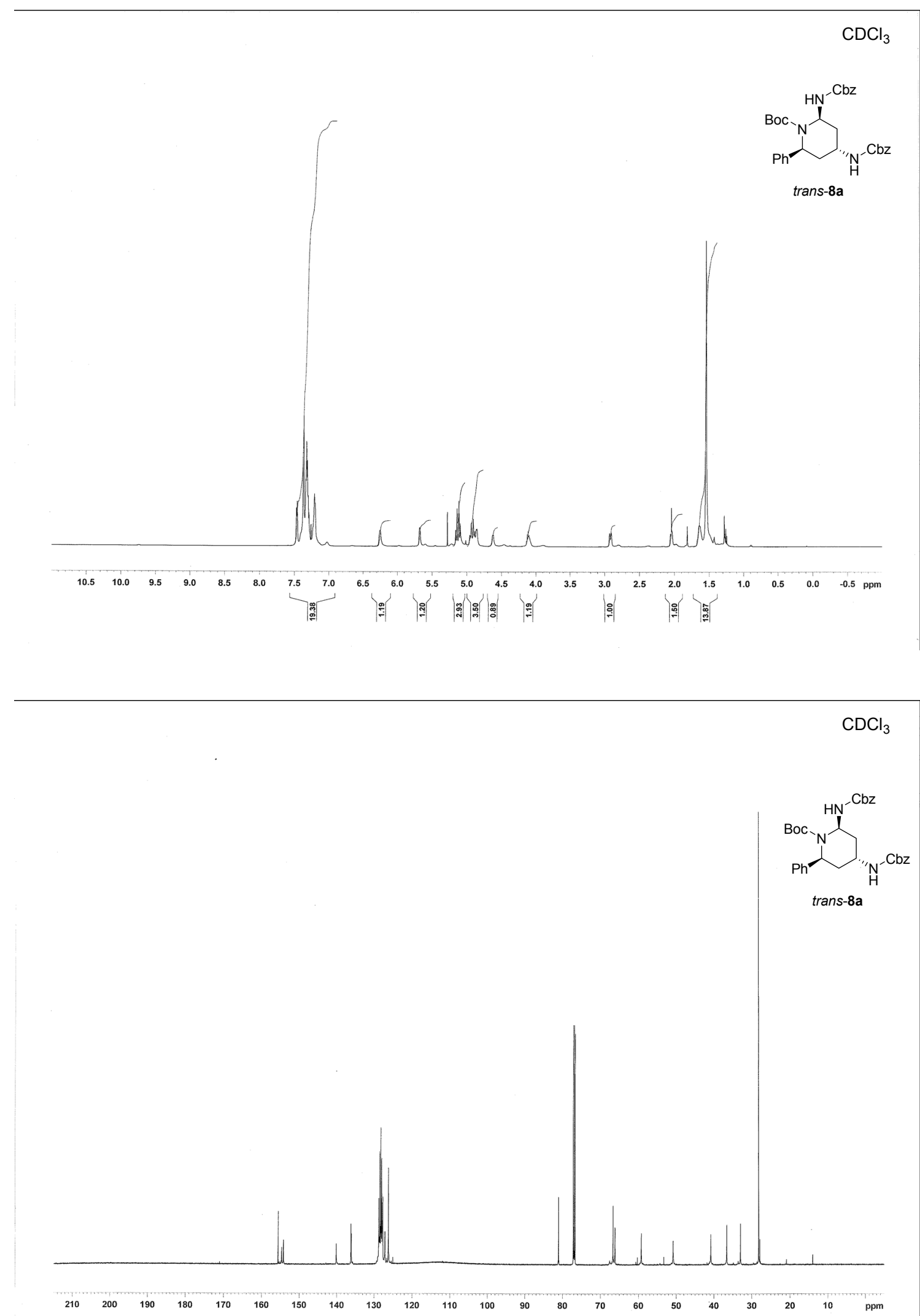


$\left(\mathrm{CD}_{3}\right)_{2} \mathrm{CO}$

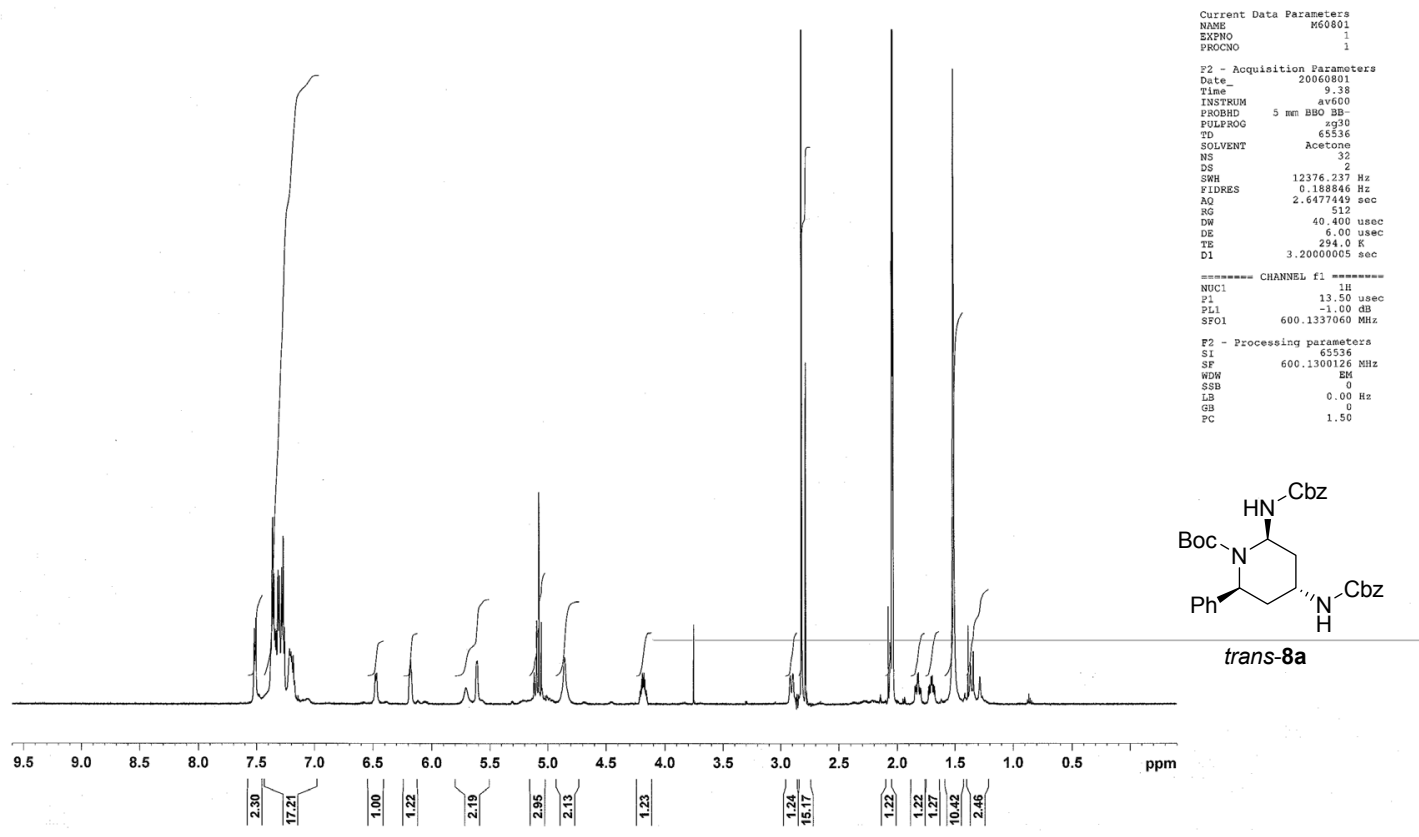

$\left(\mathrm{CD}_{3}\right)_{2} \mathrm{CO}$
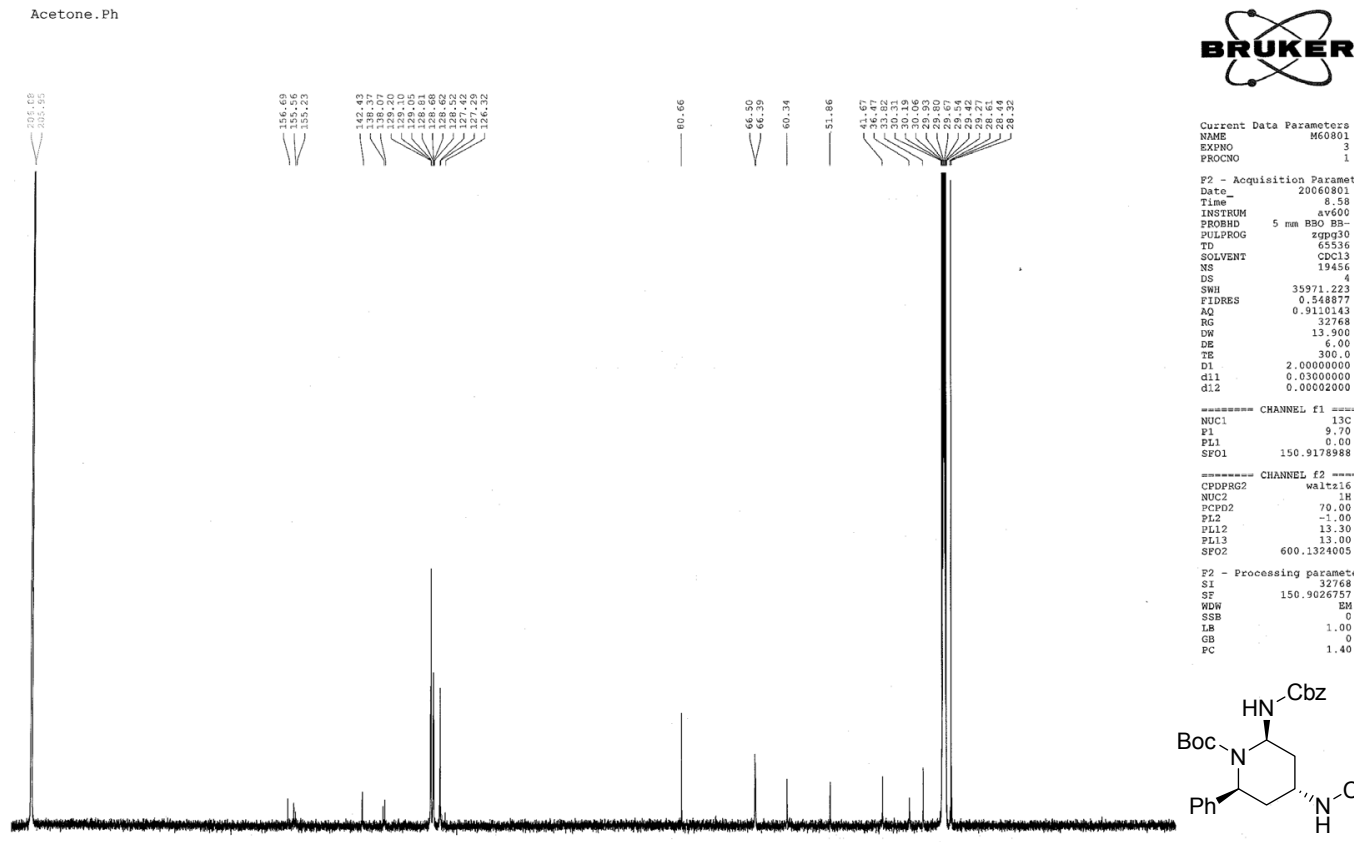

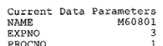

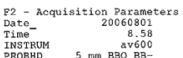

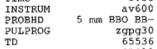

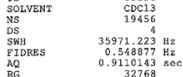

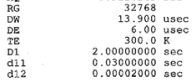

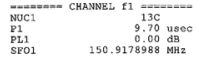

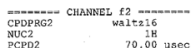

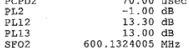

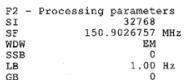

trans-8a

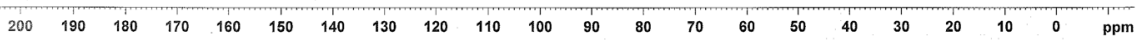



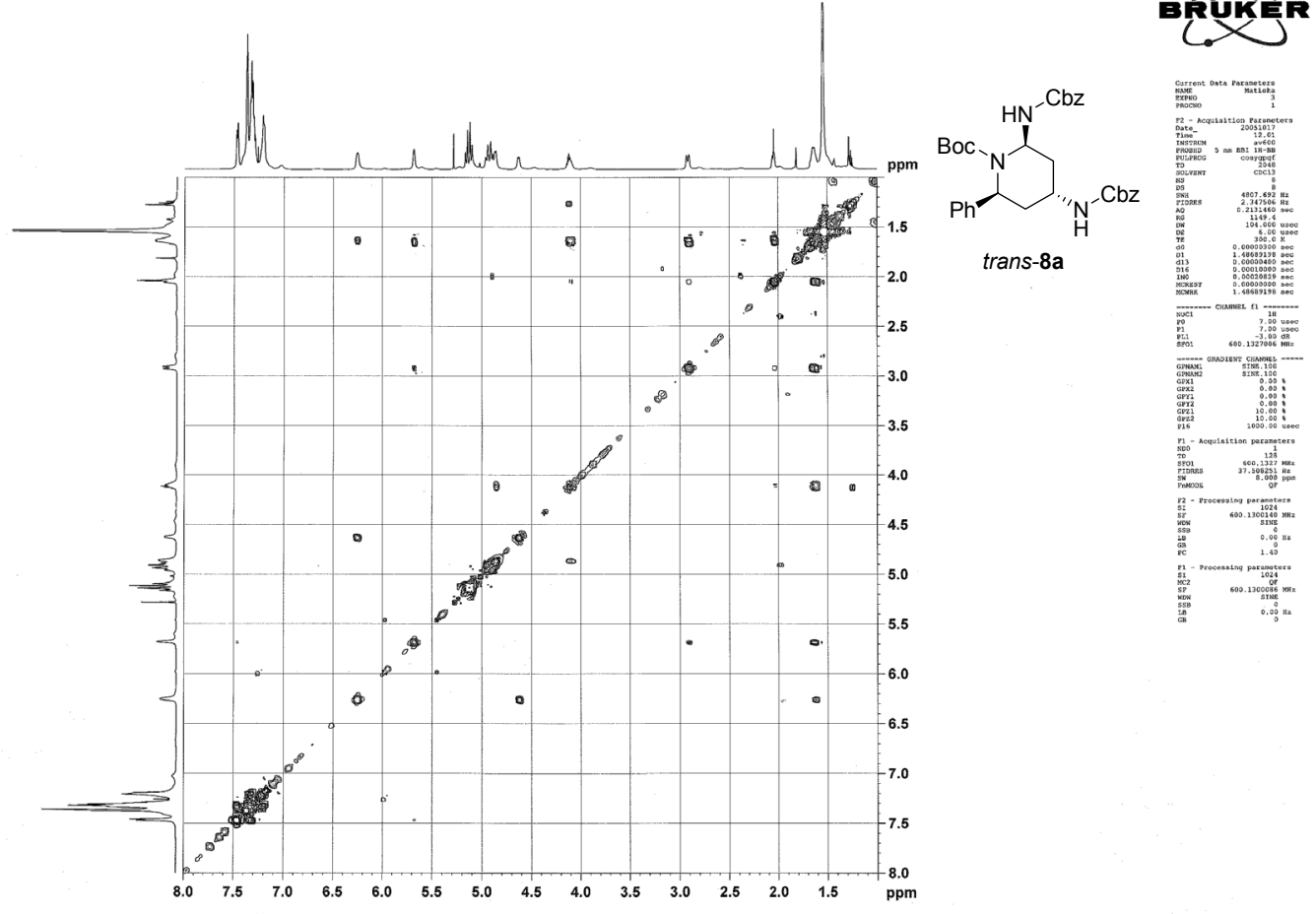

$\left(\mathrm{CD}_{3}\right)_{2} \mathrm{CO}$

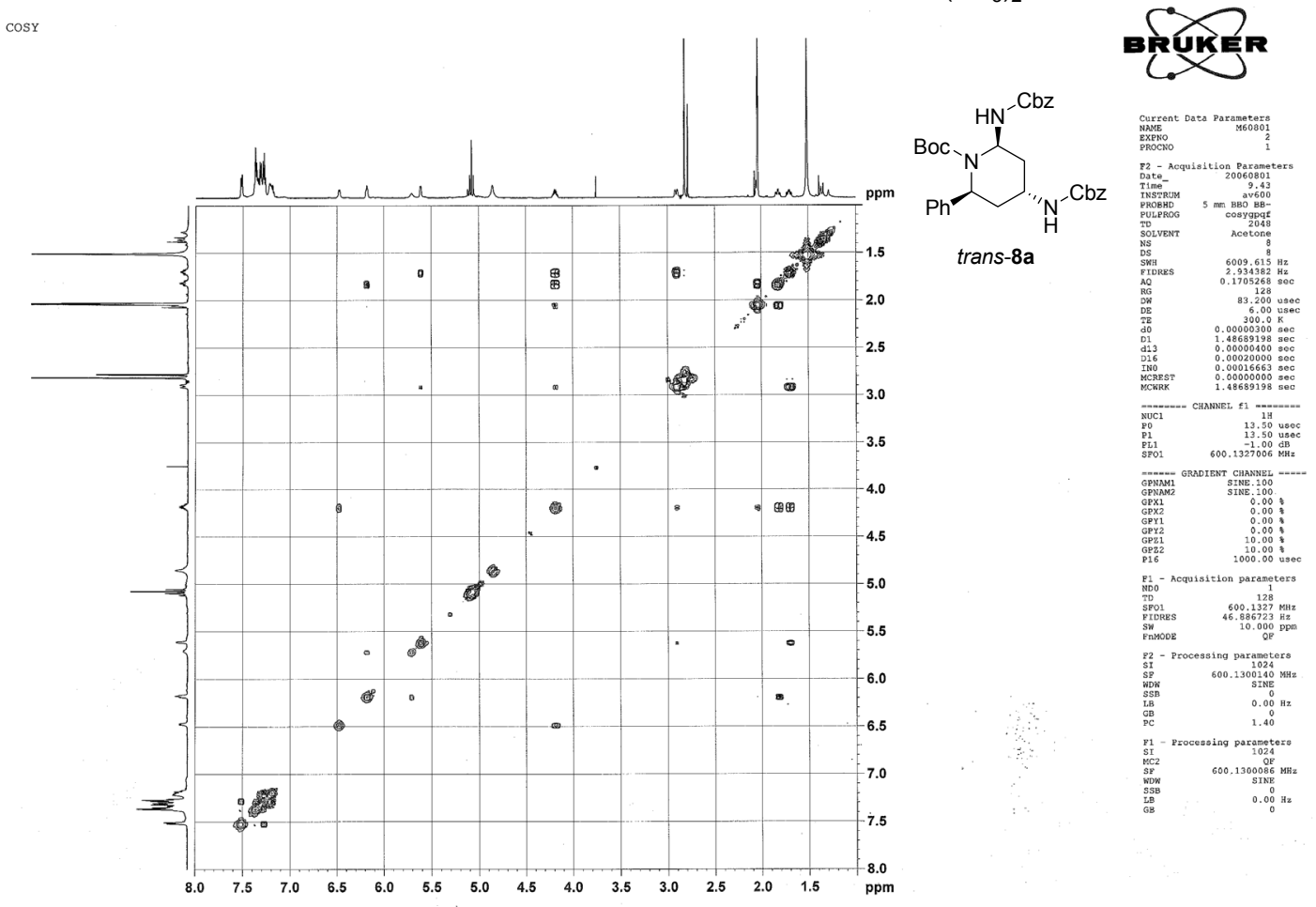




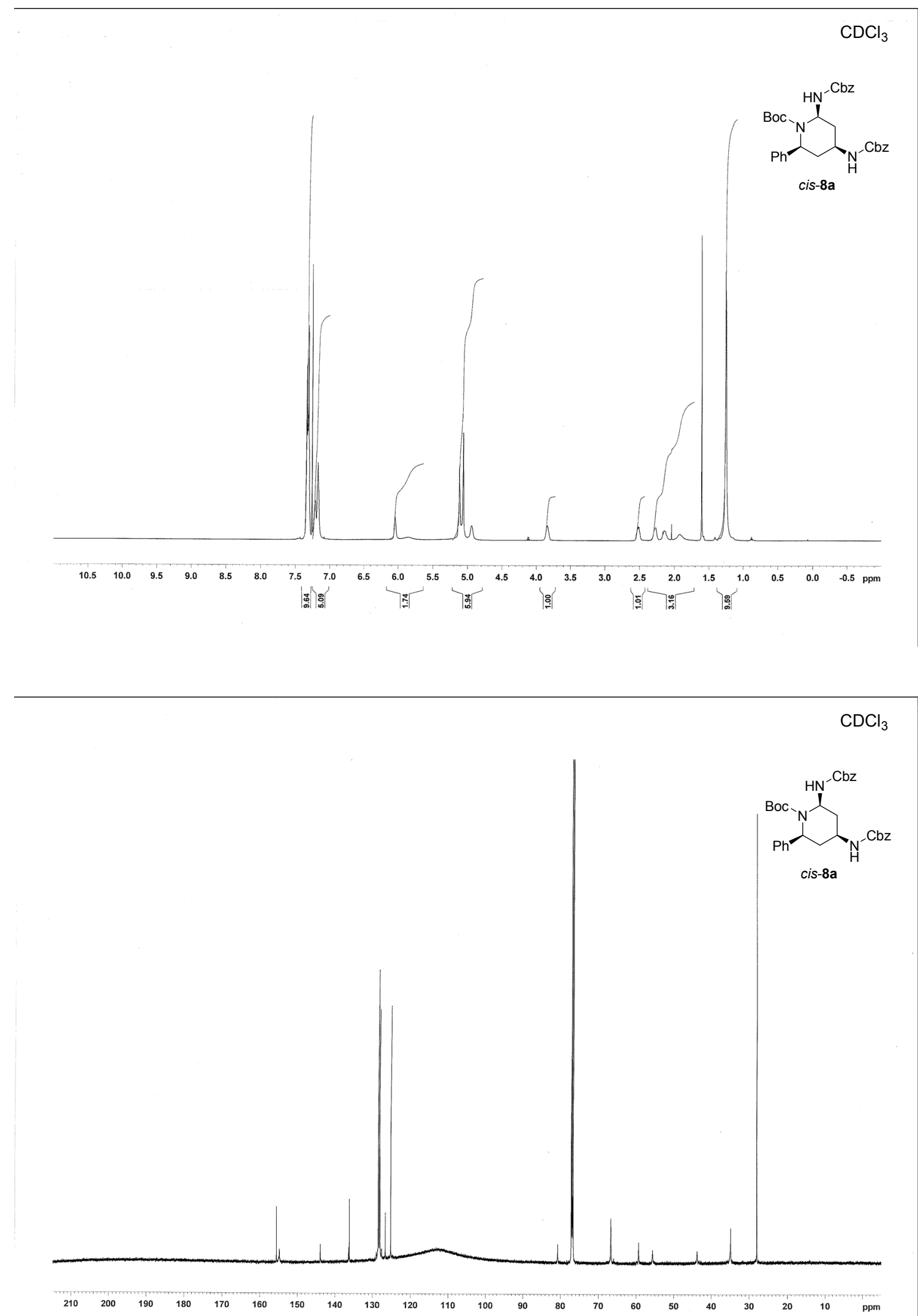



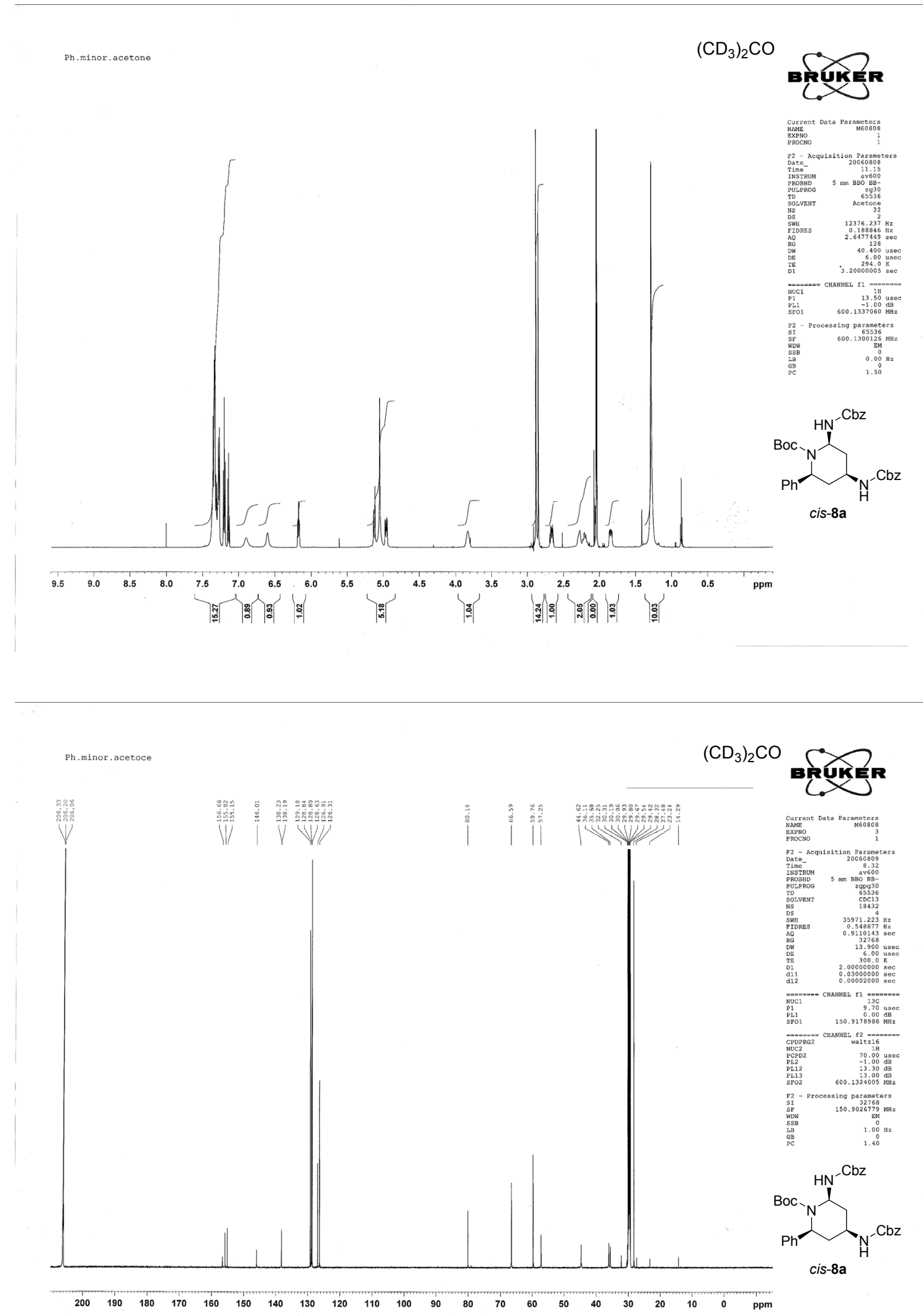

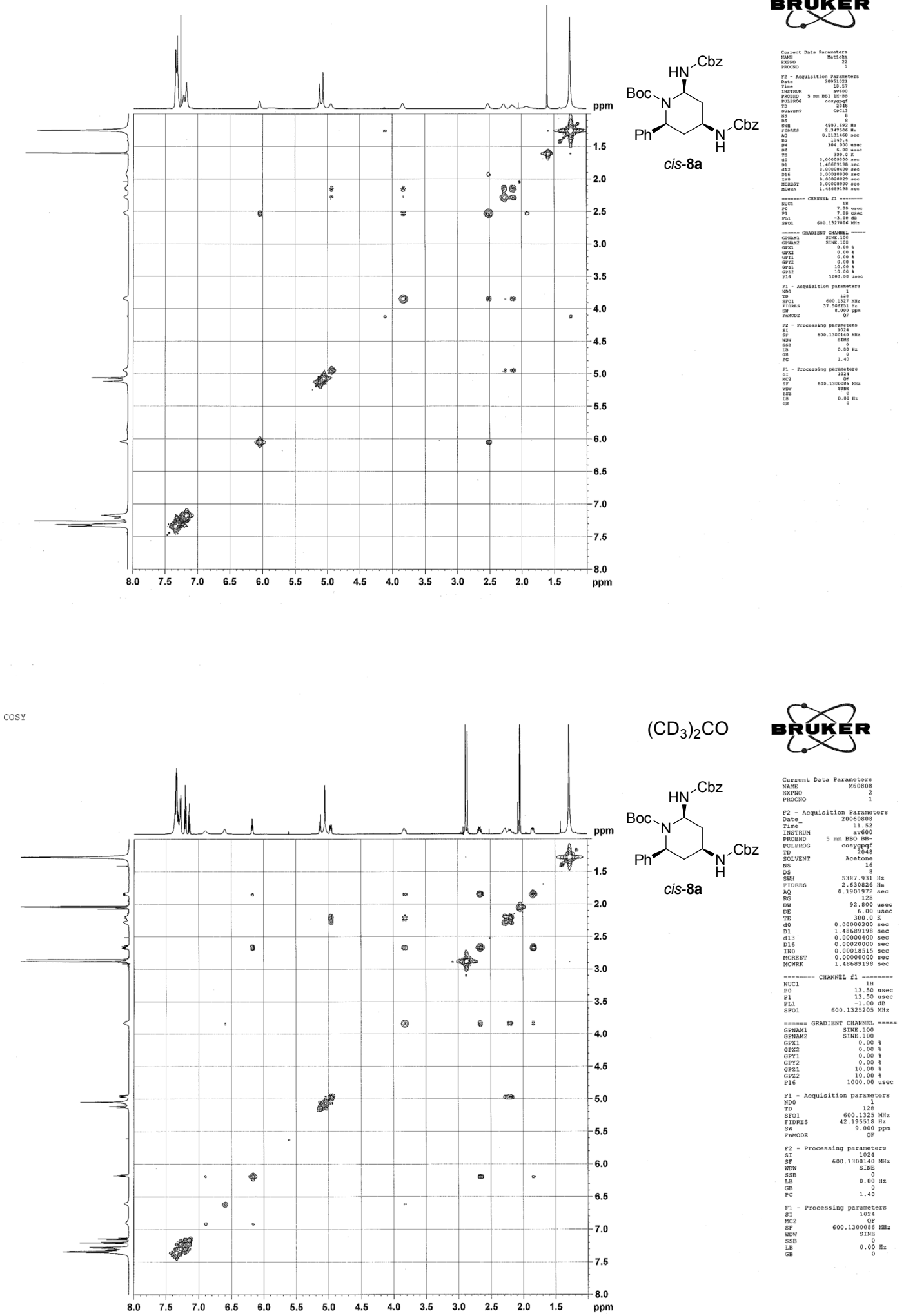


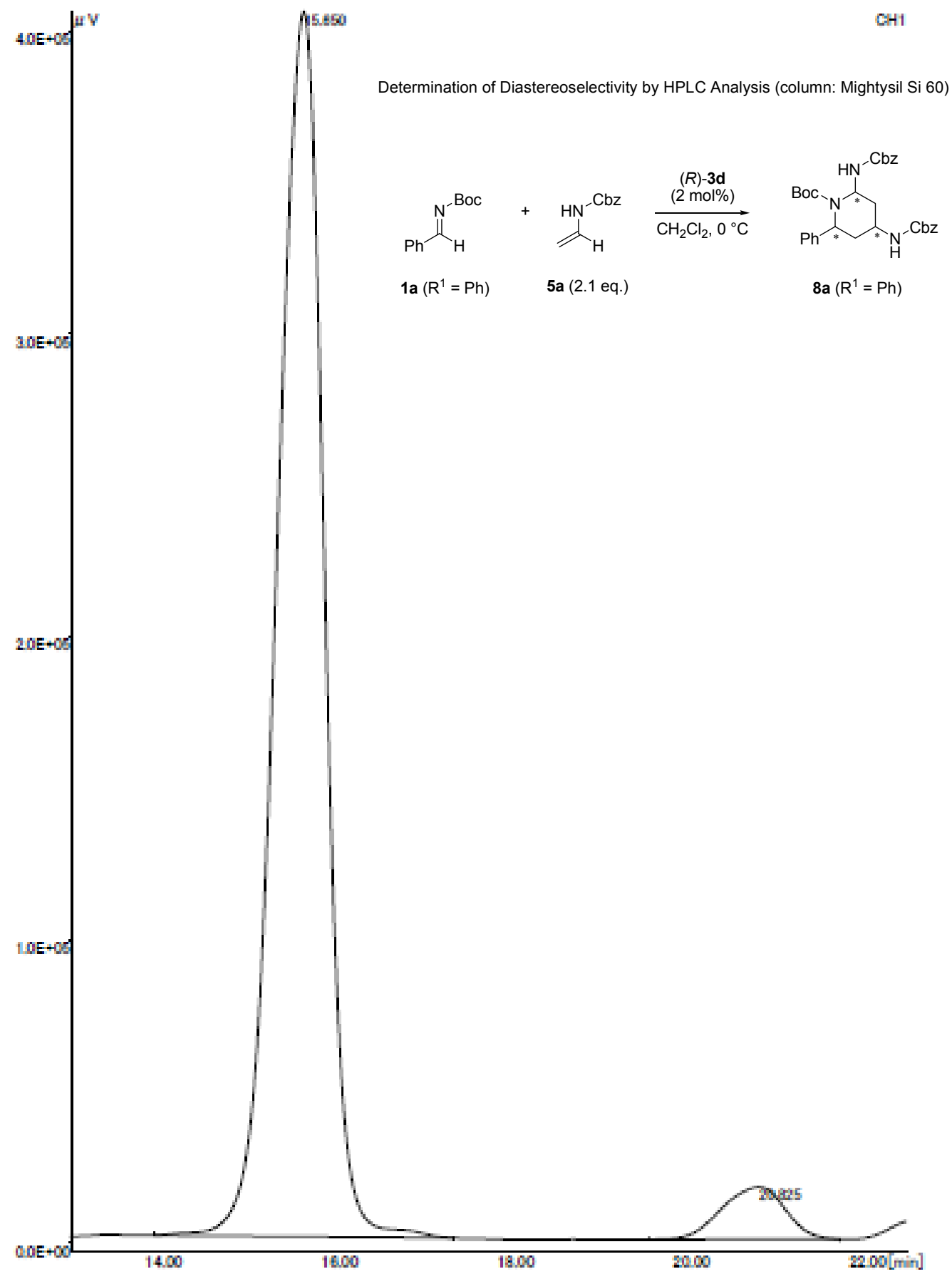




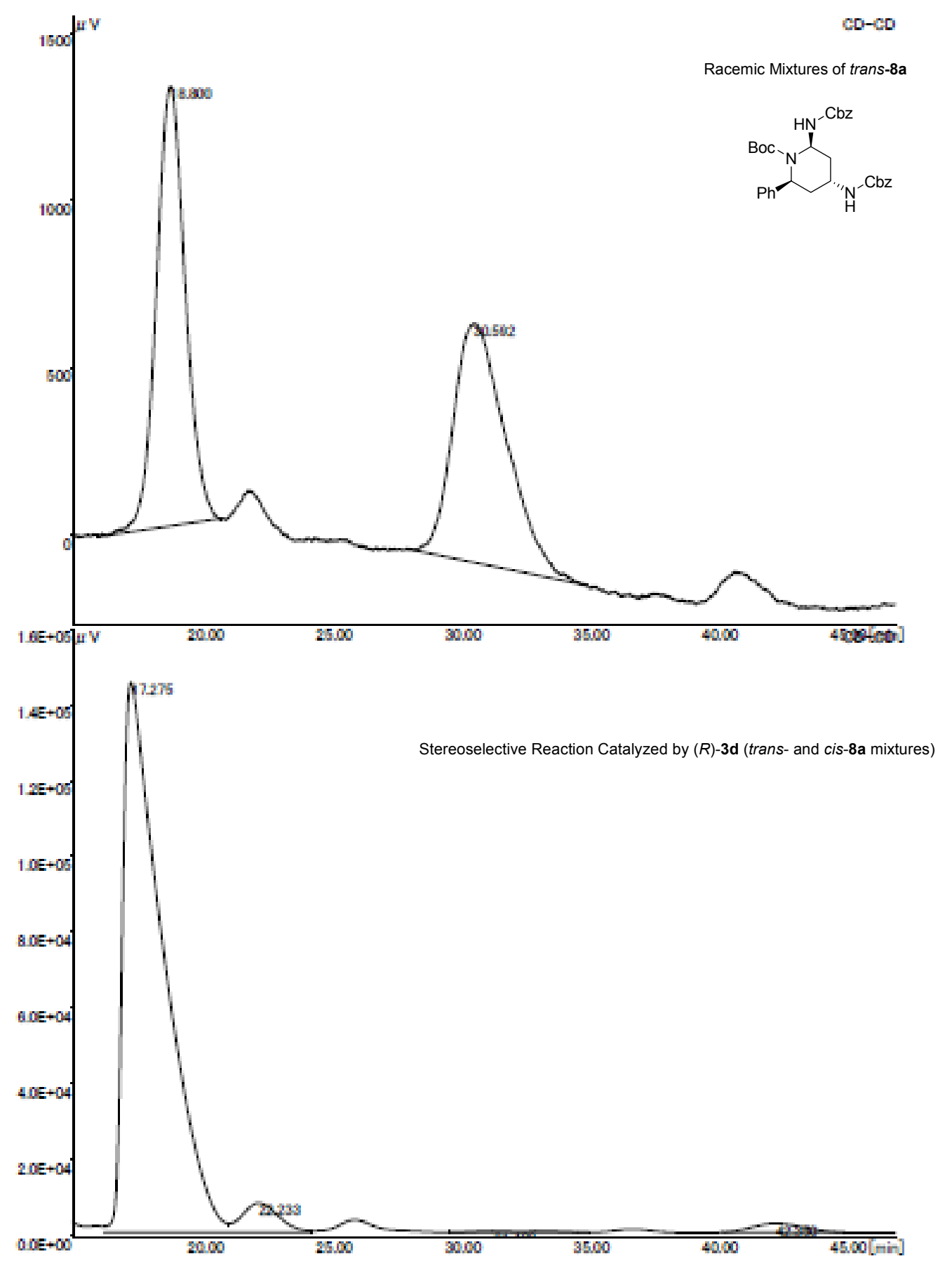




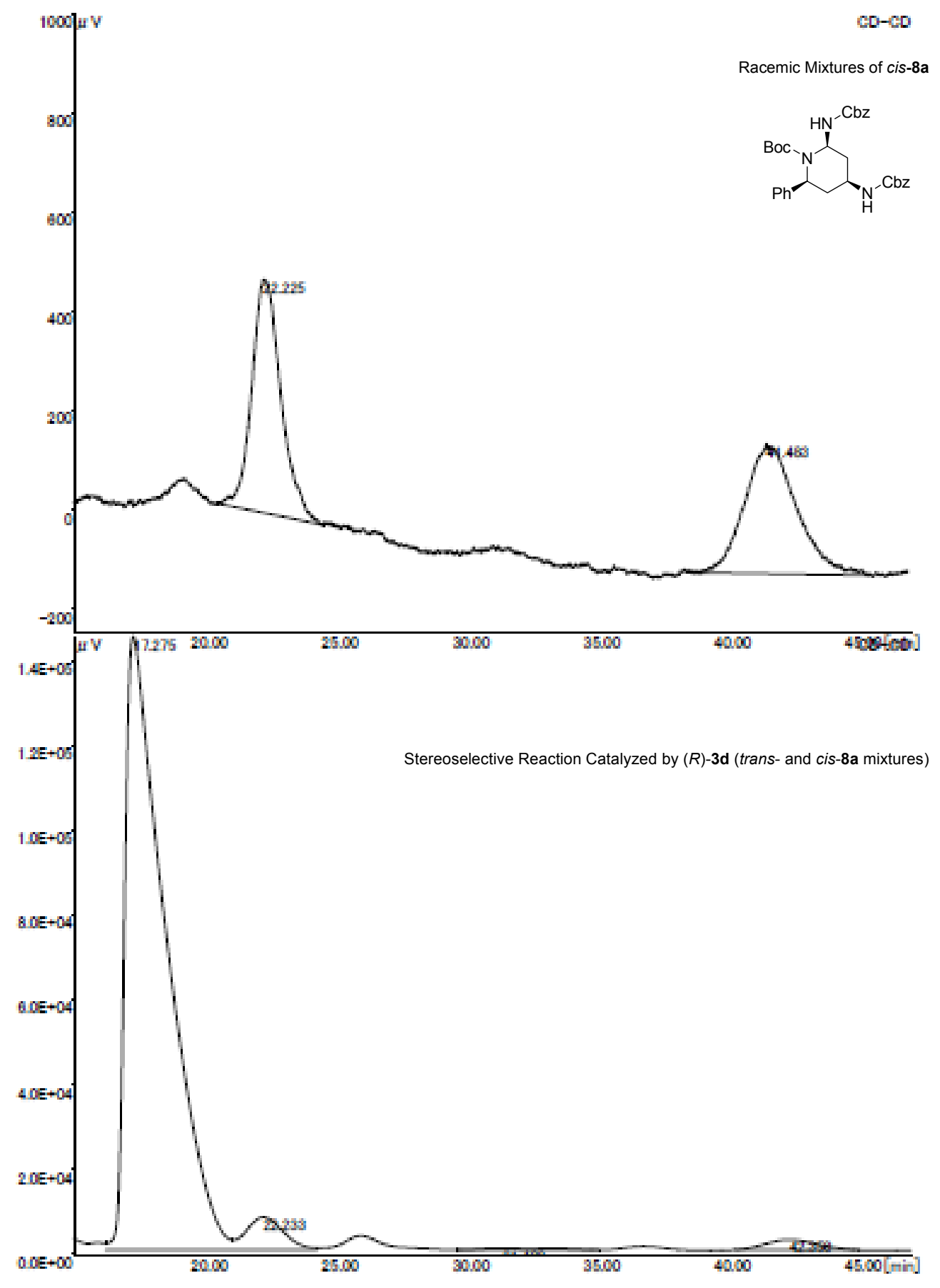




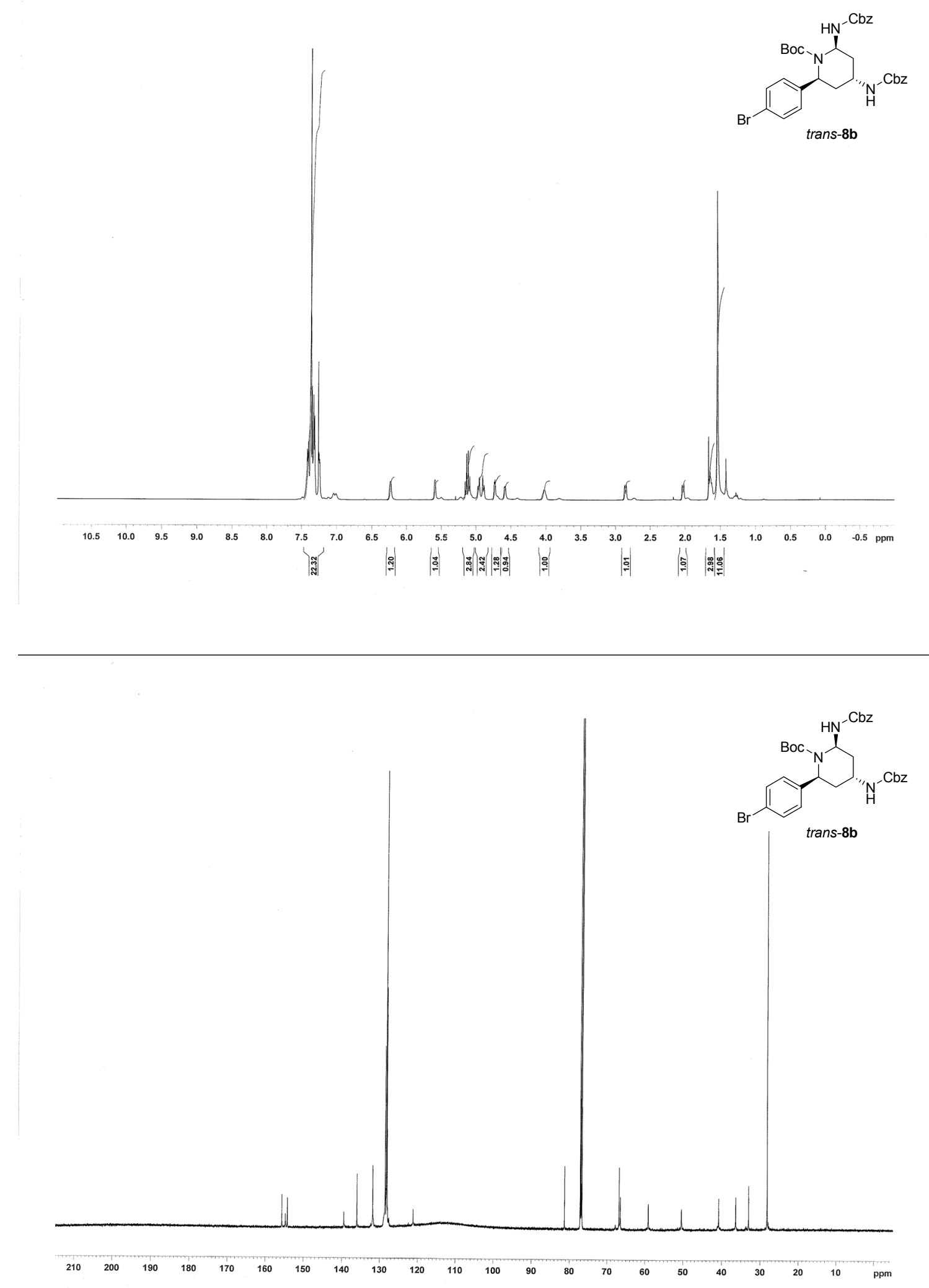



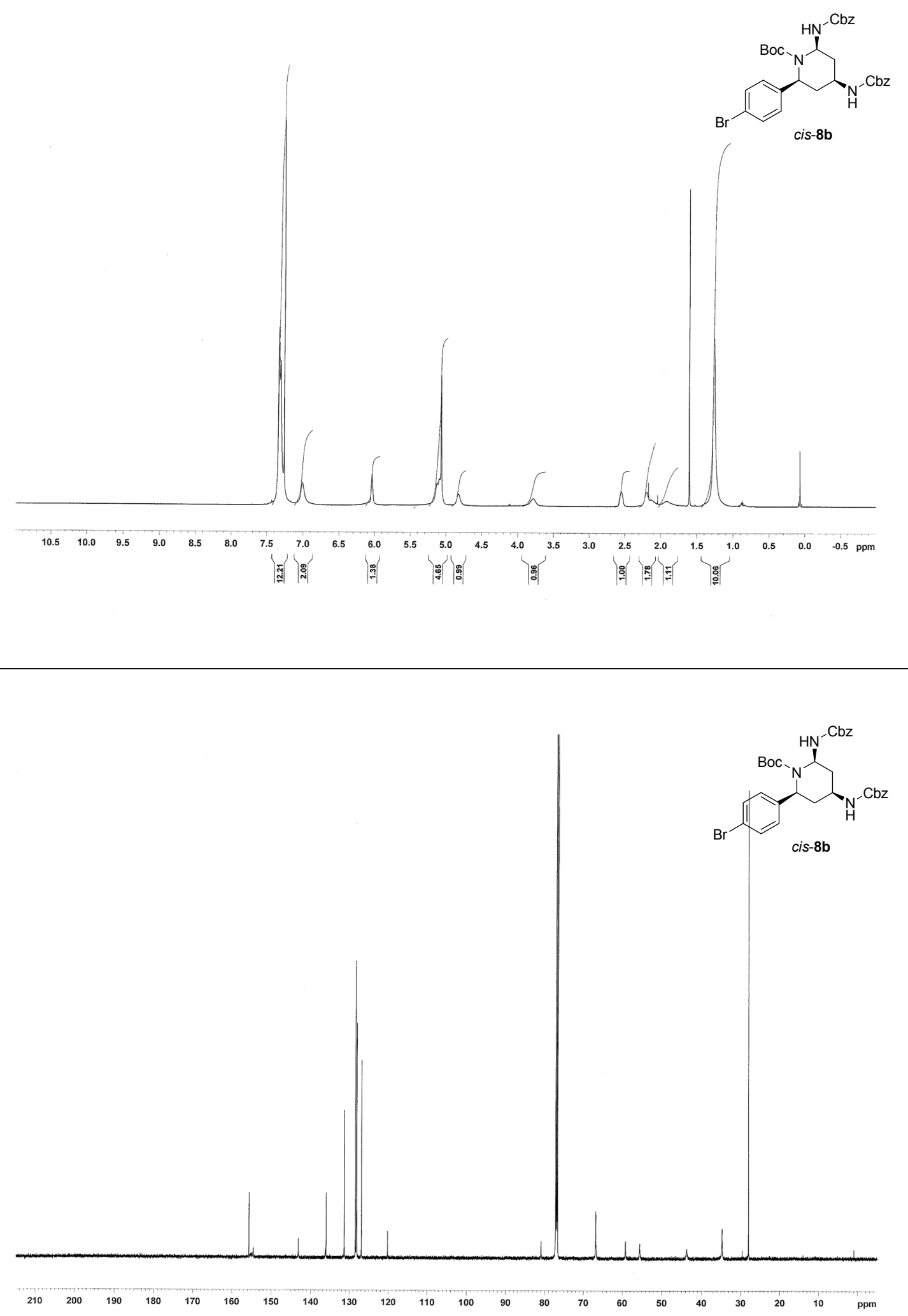


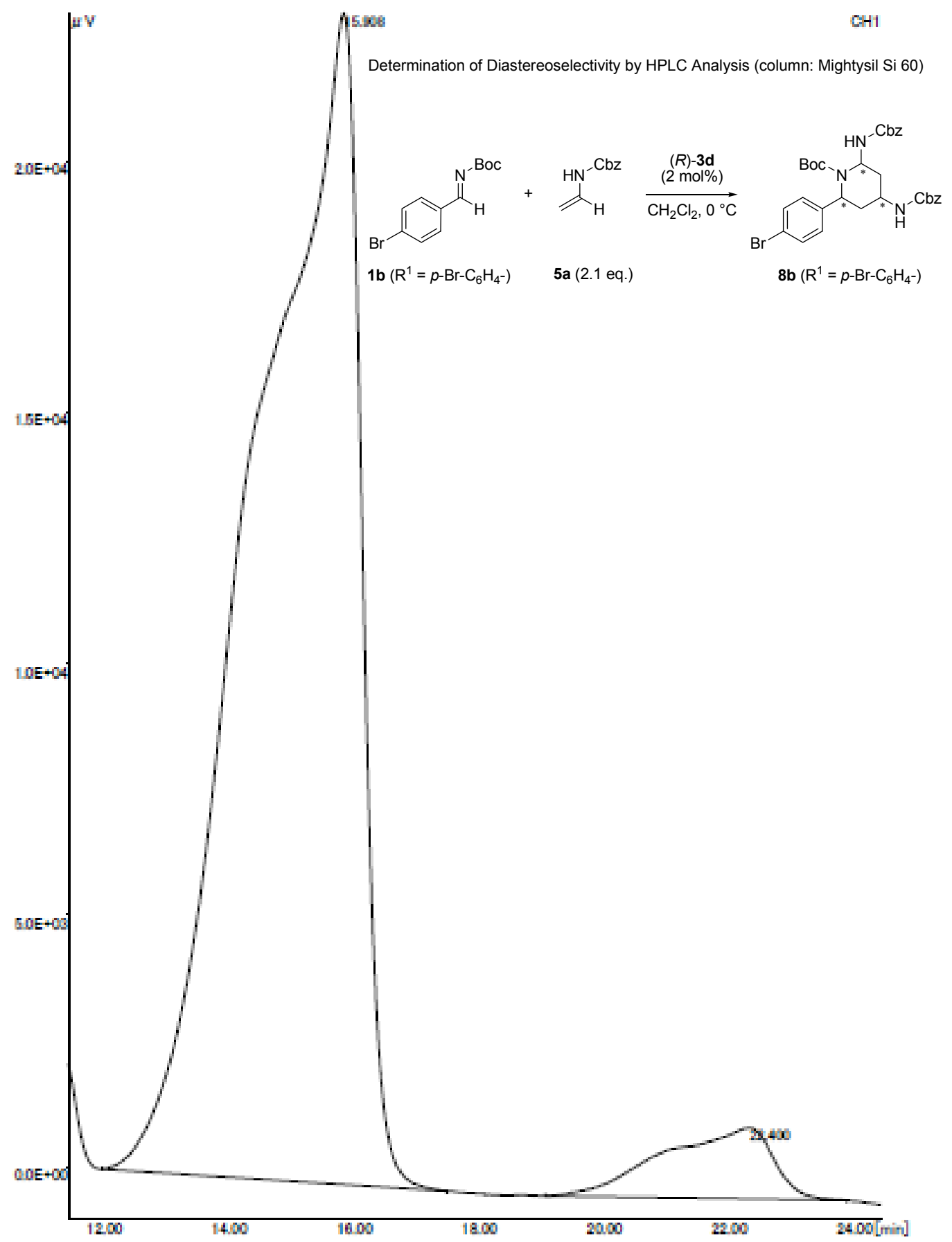




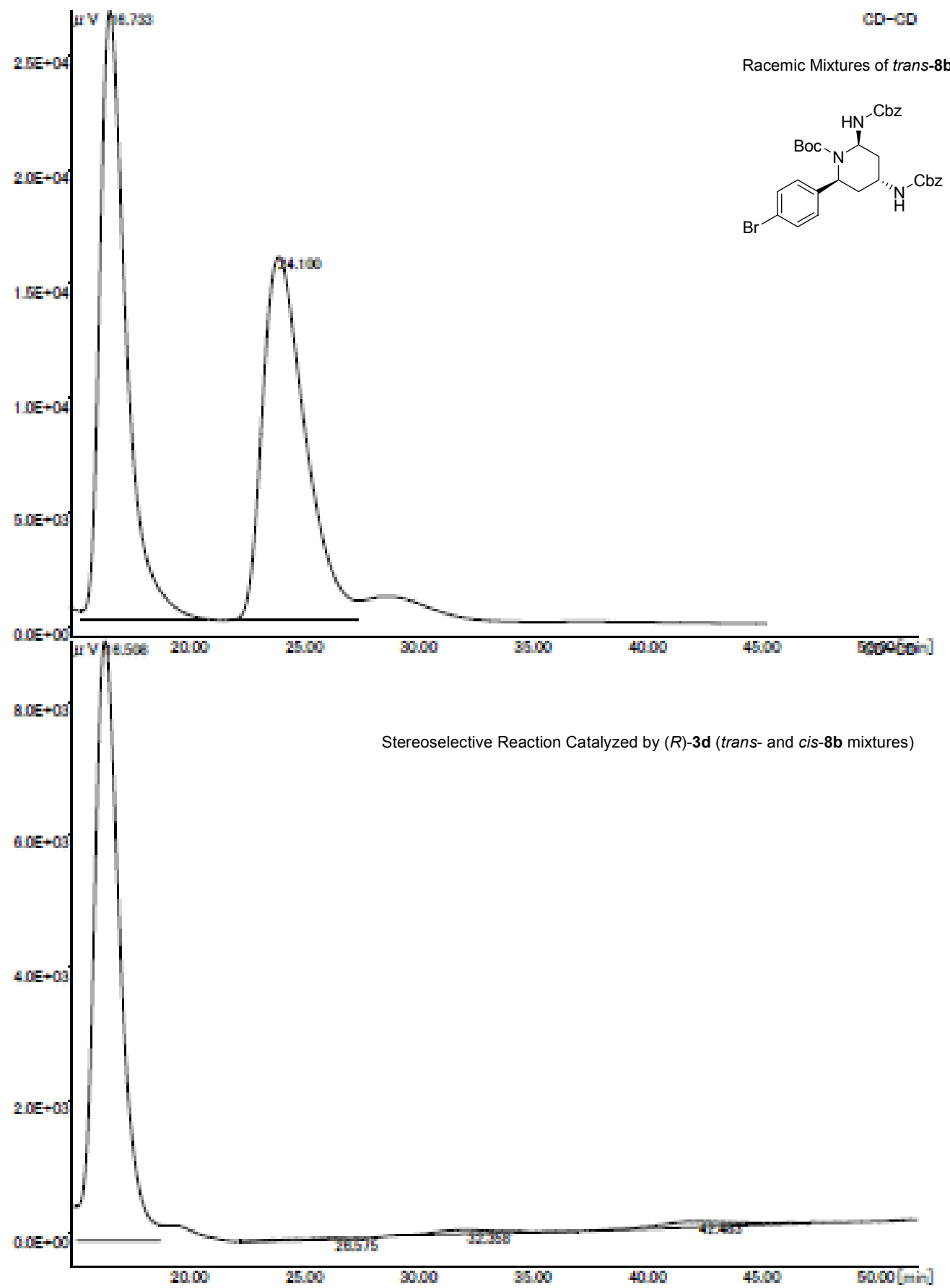




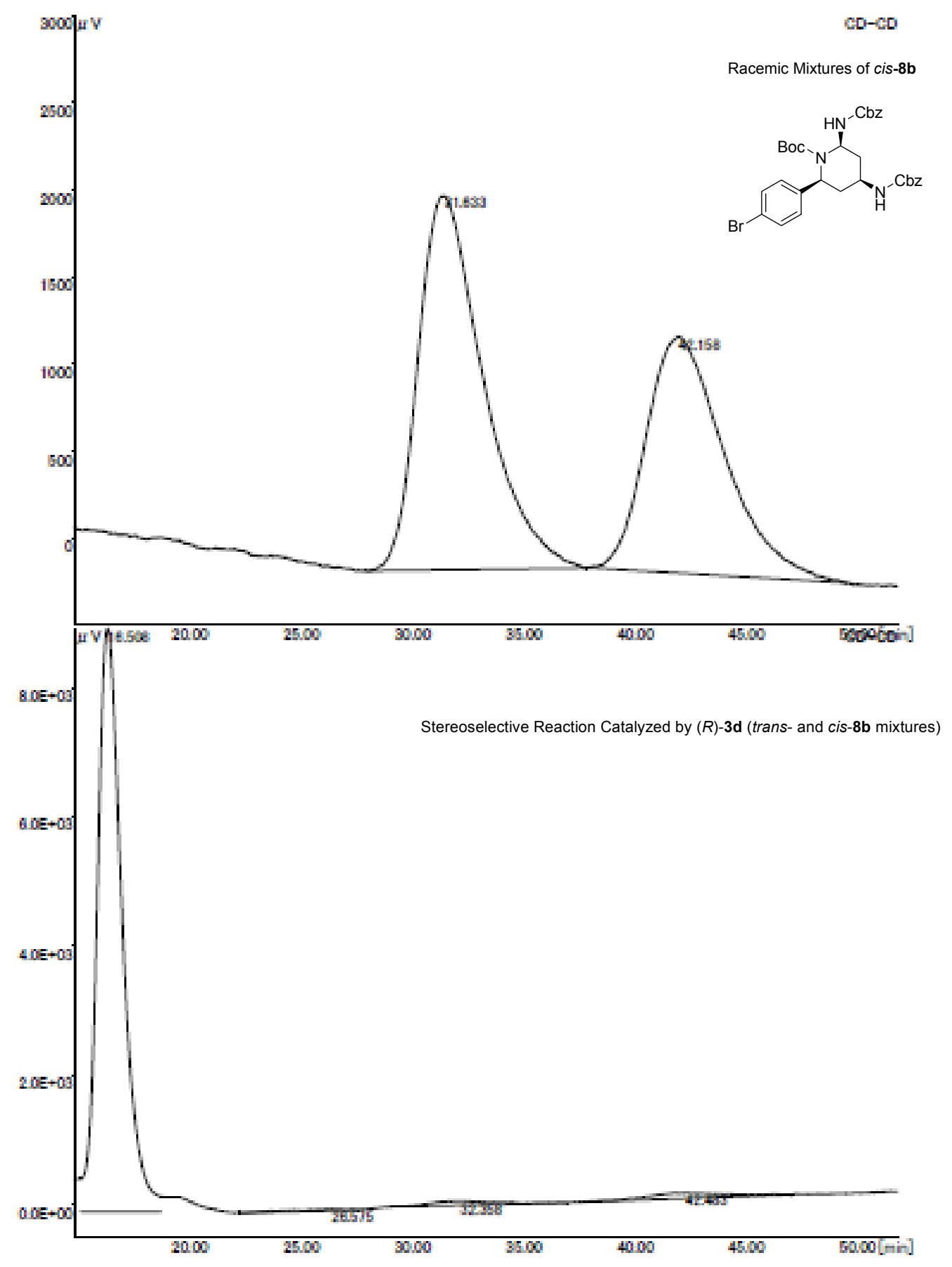




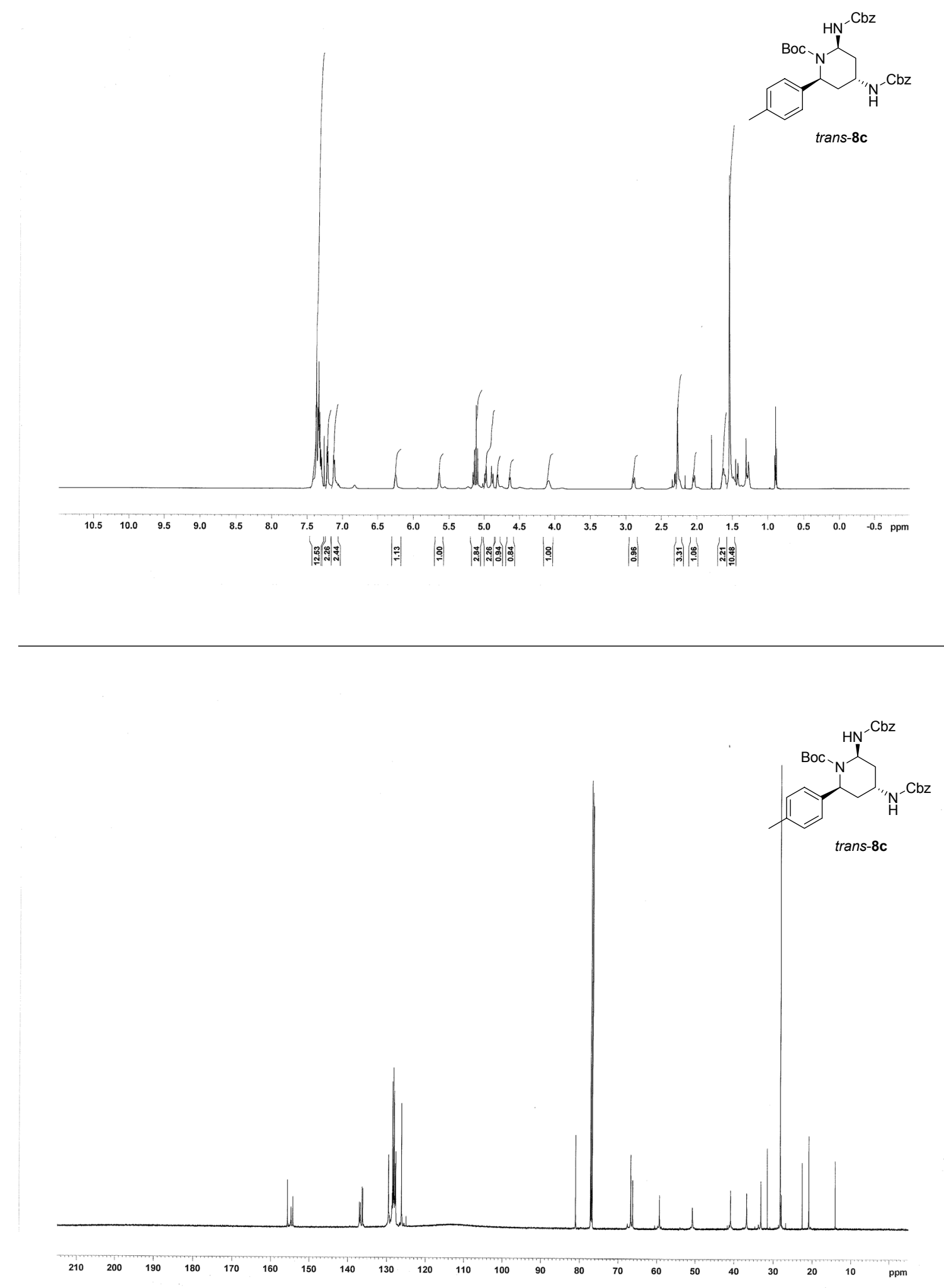



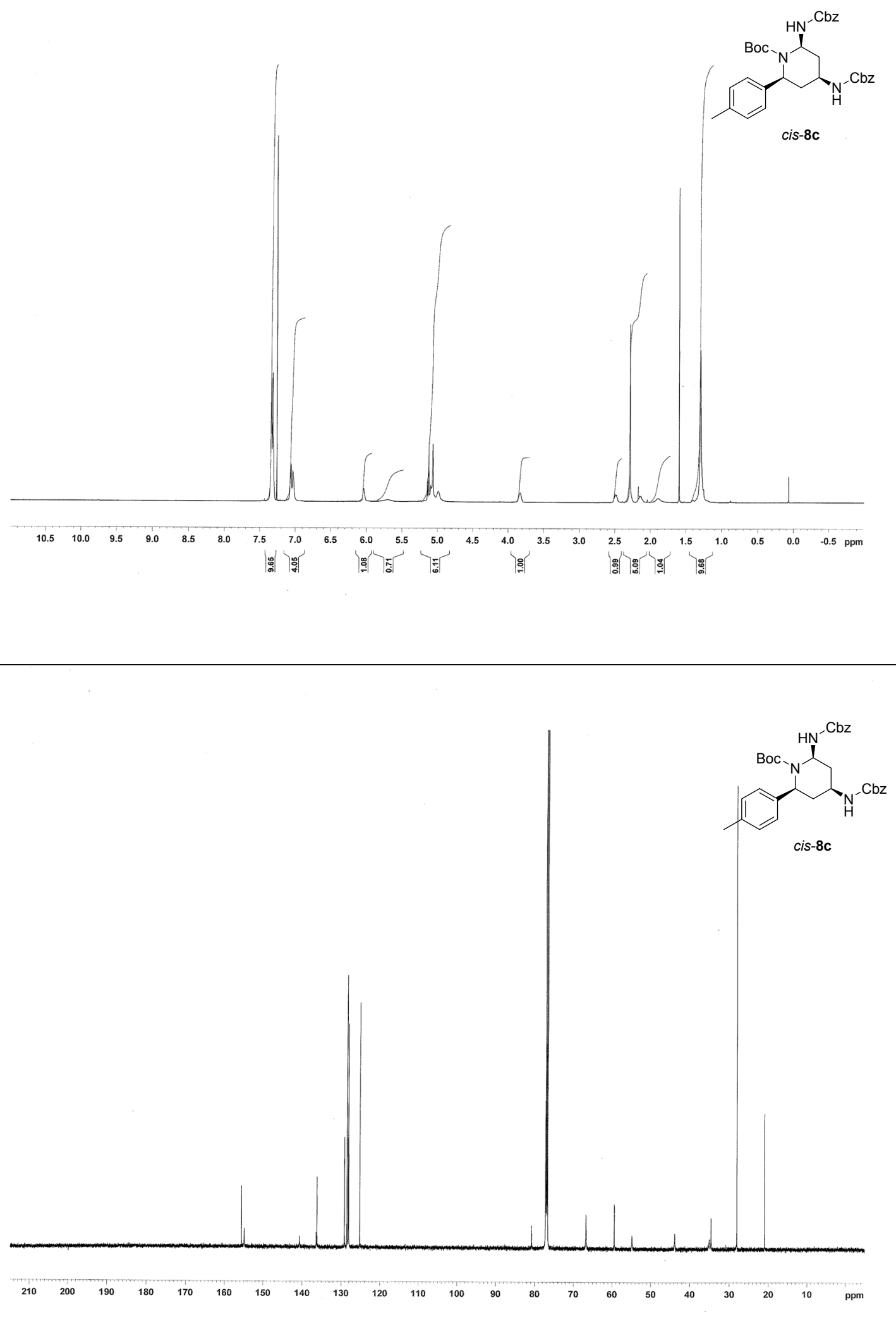

S26 


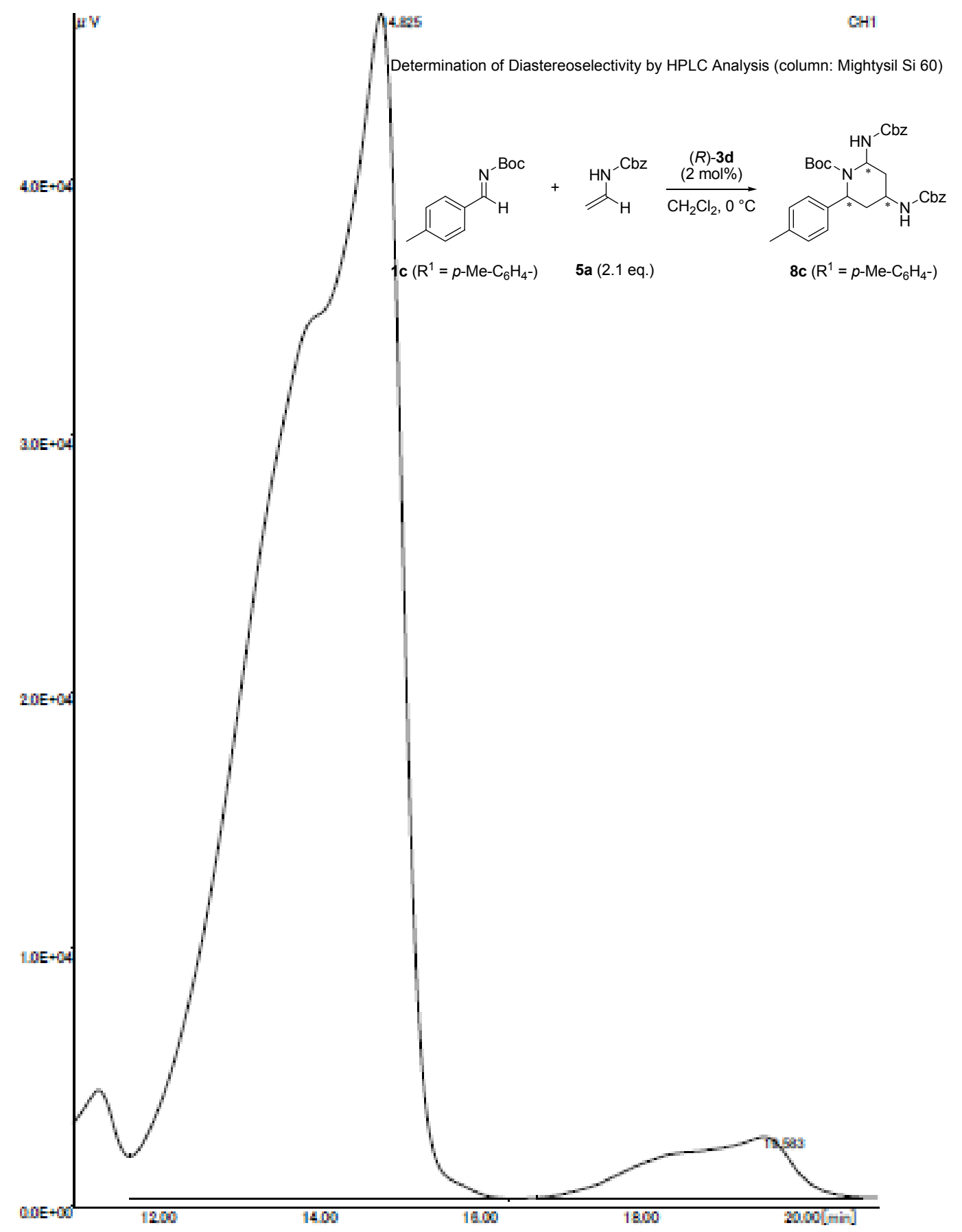




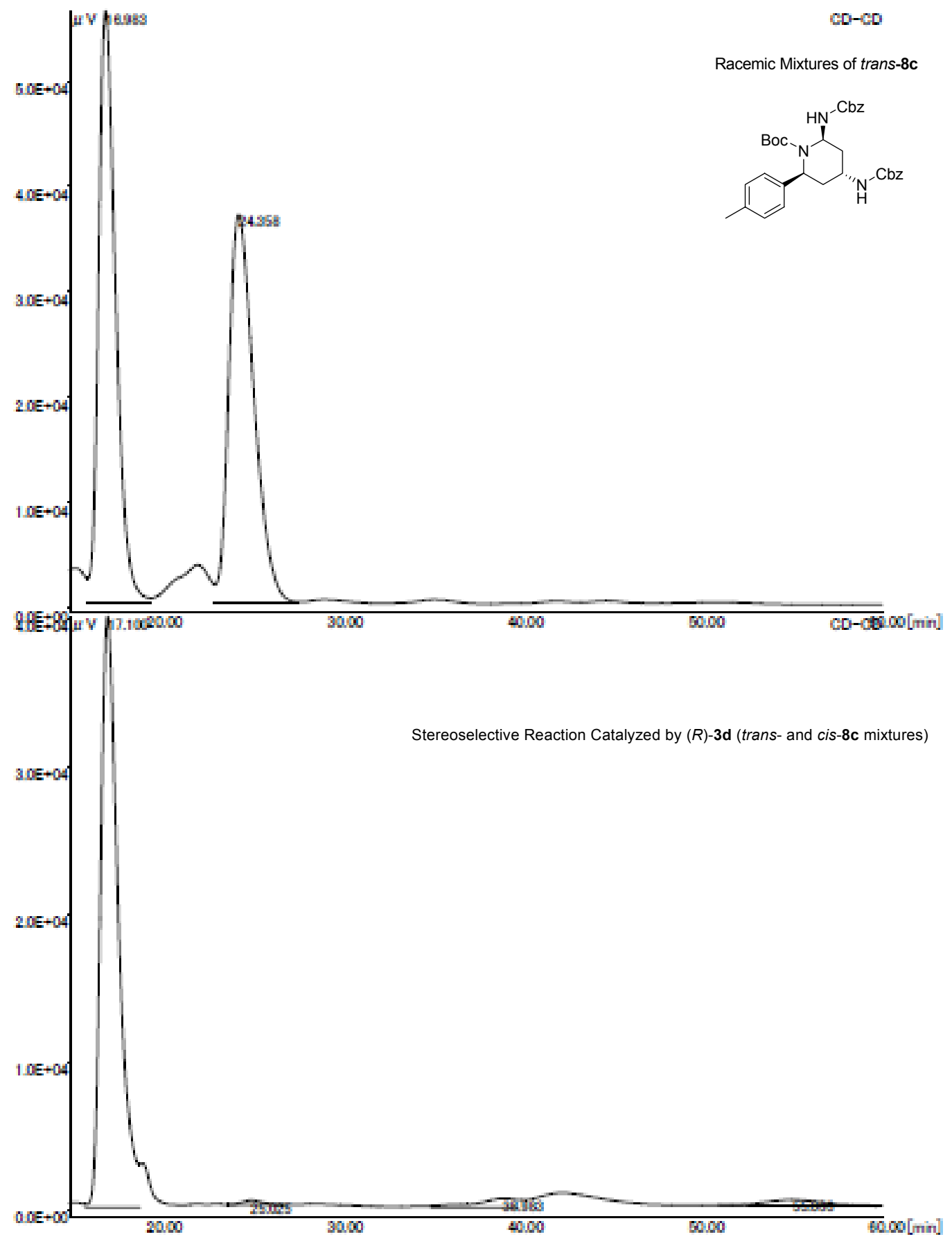




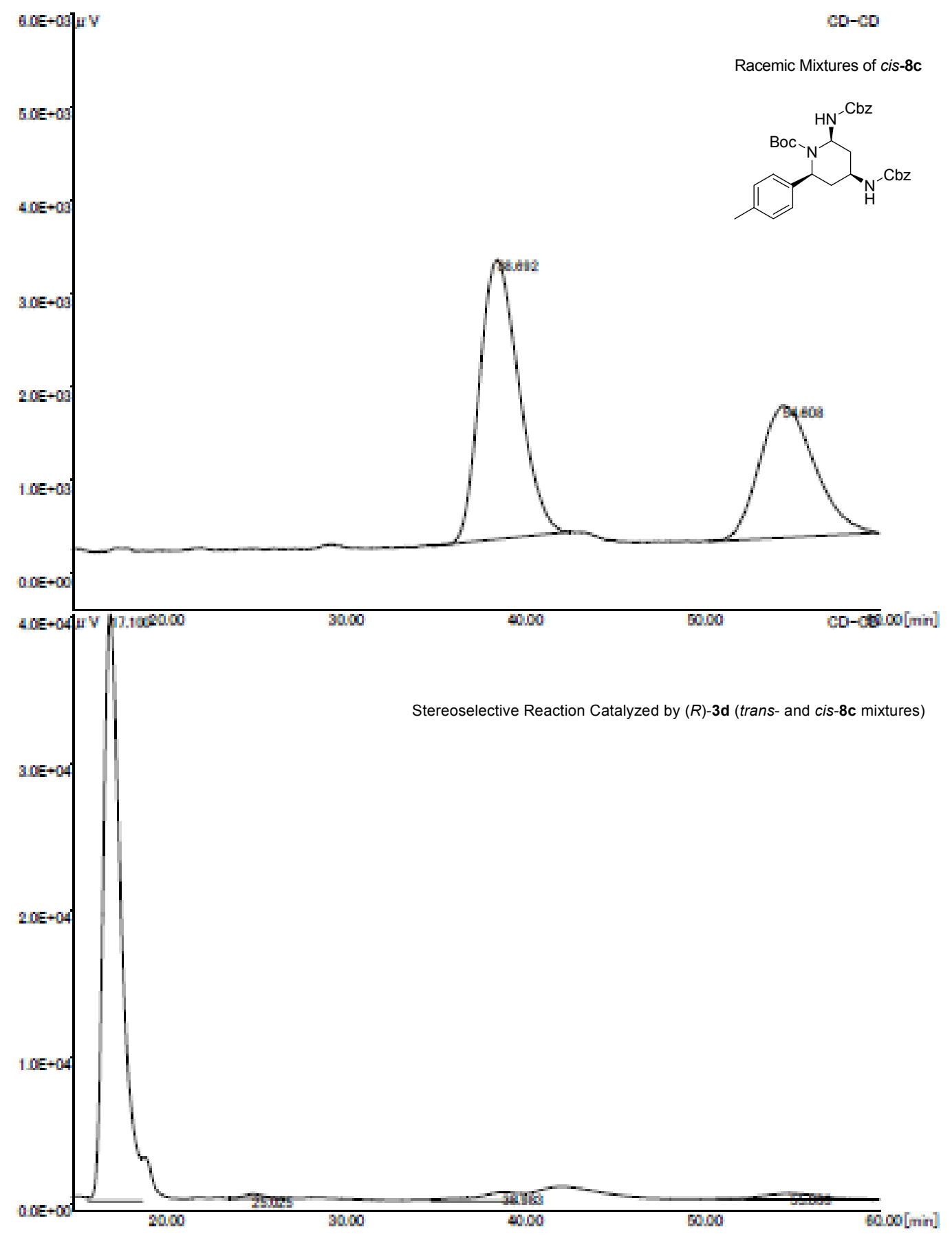



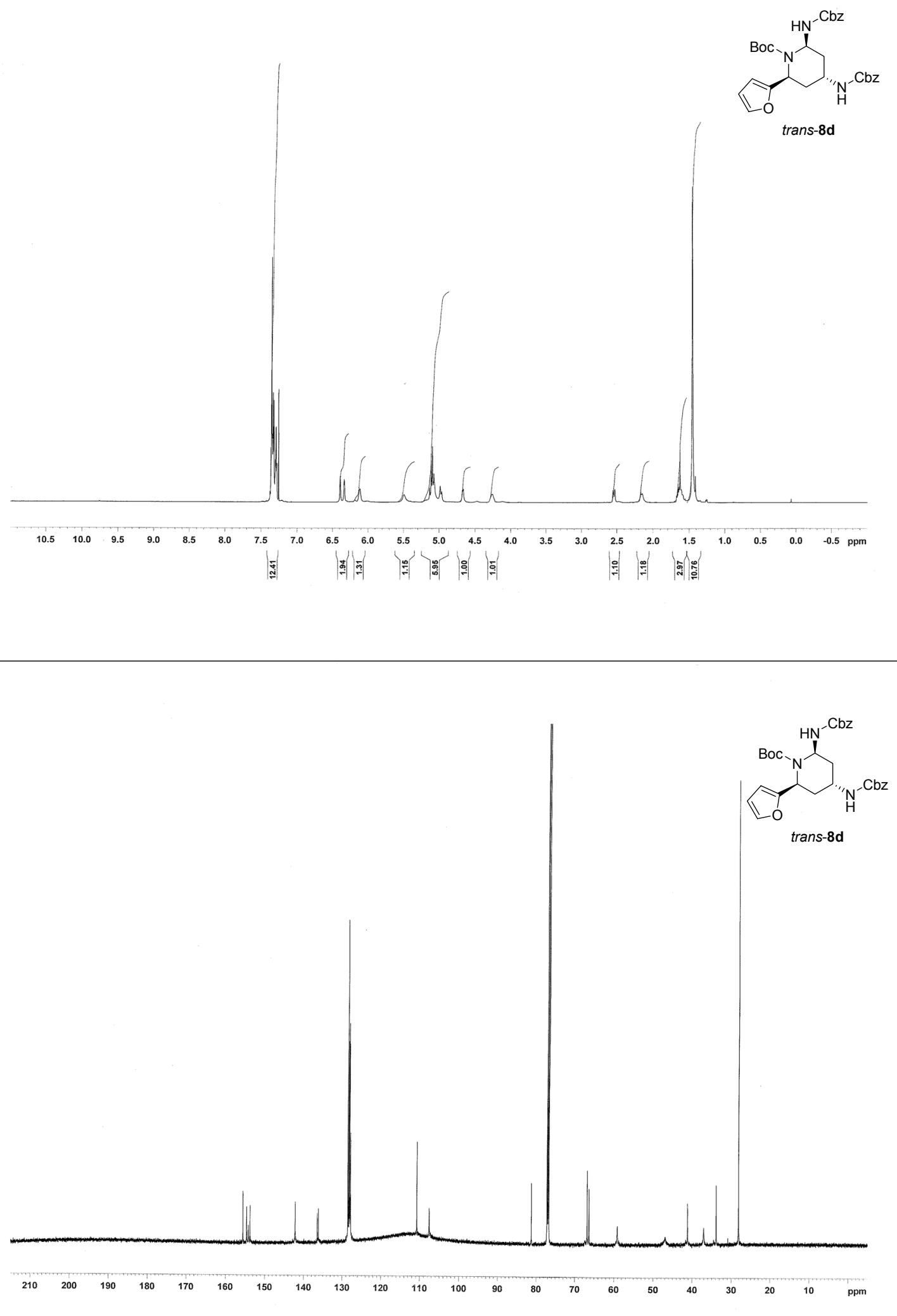

S30 

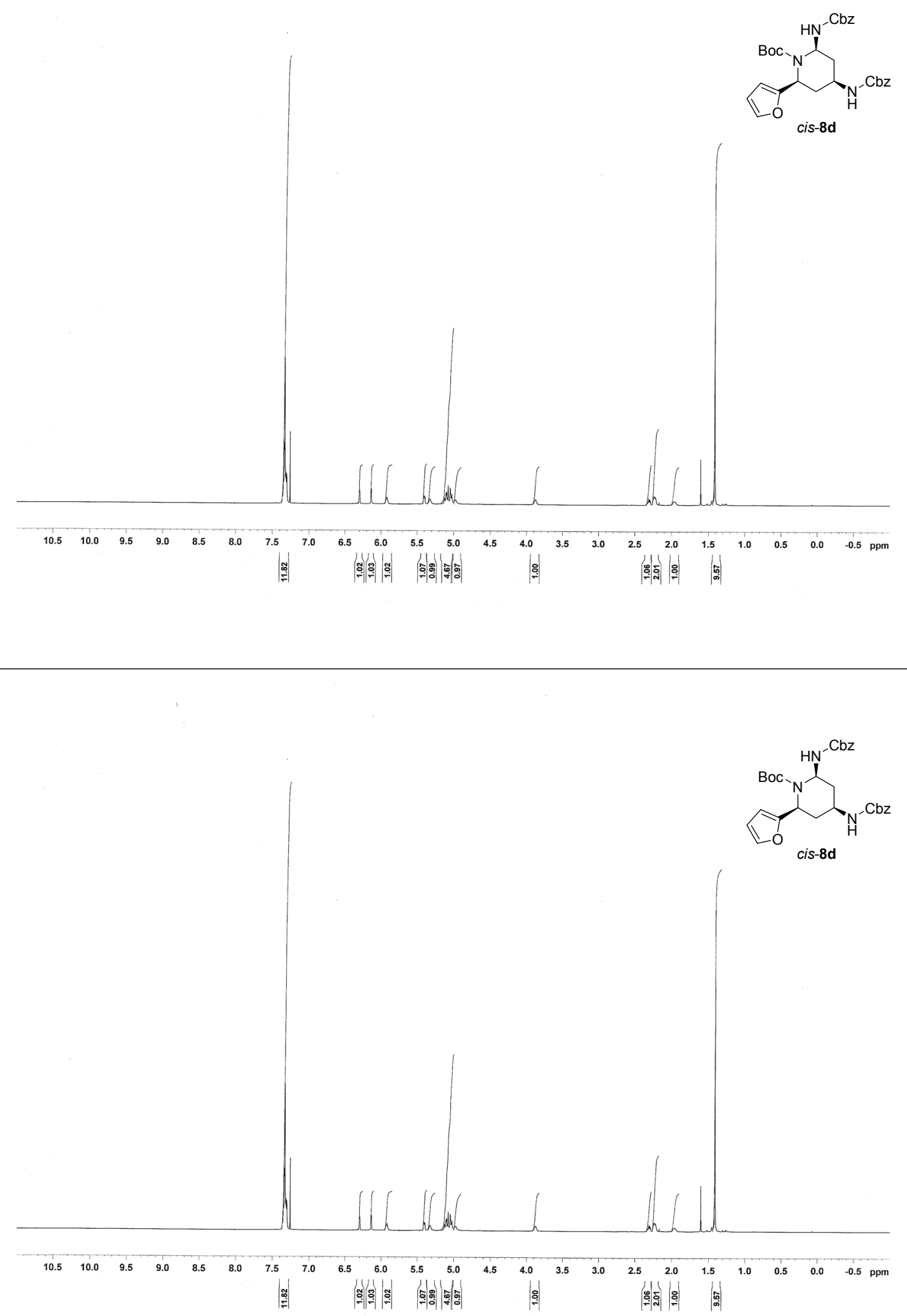


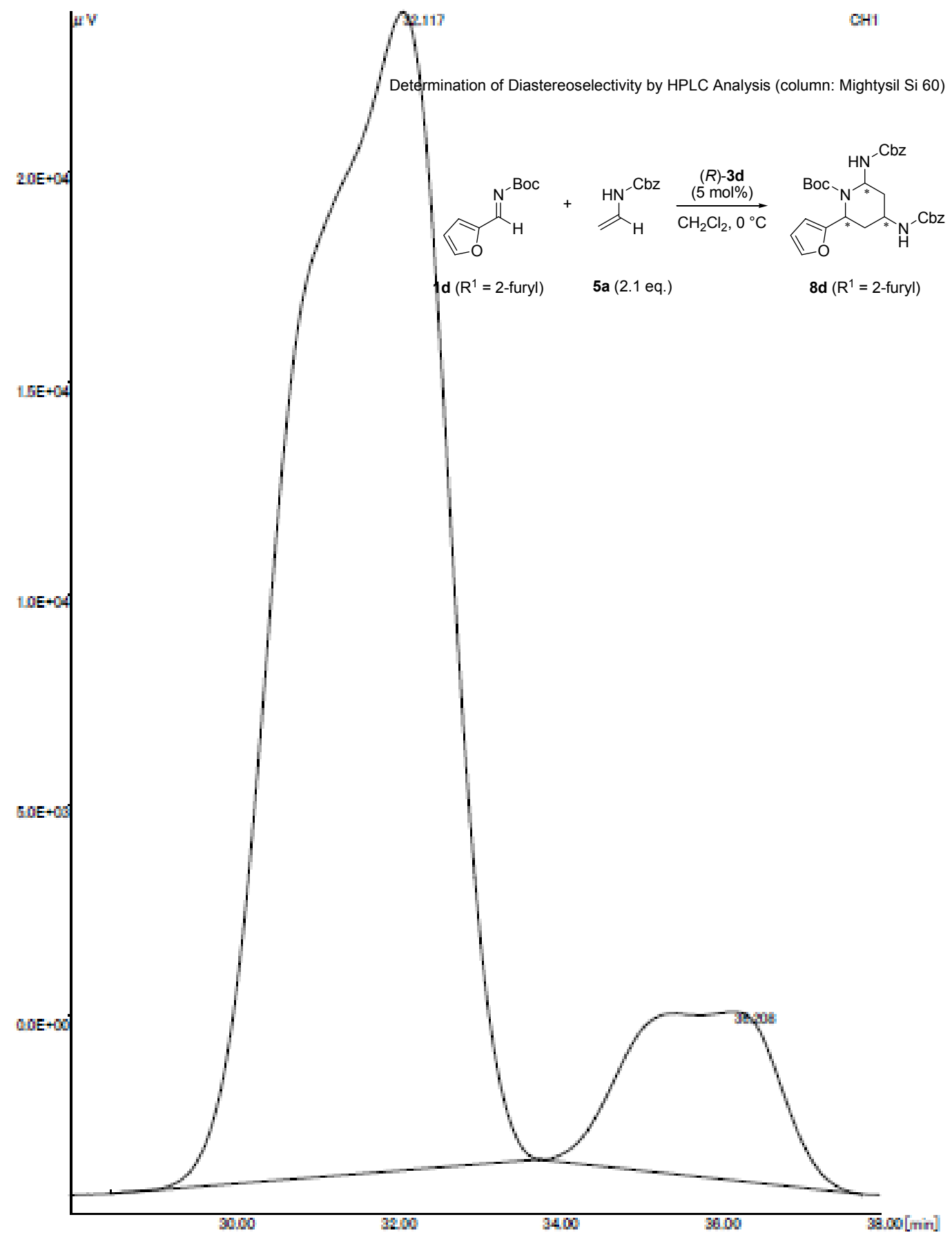




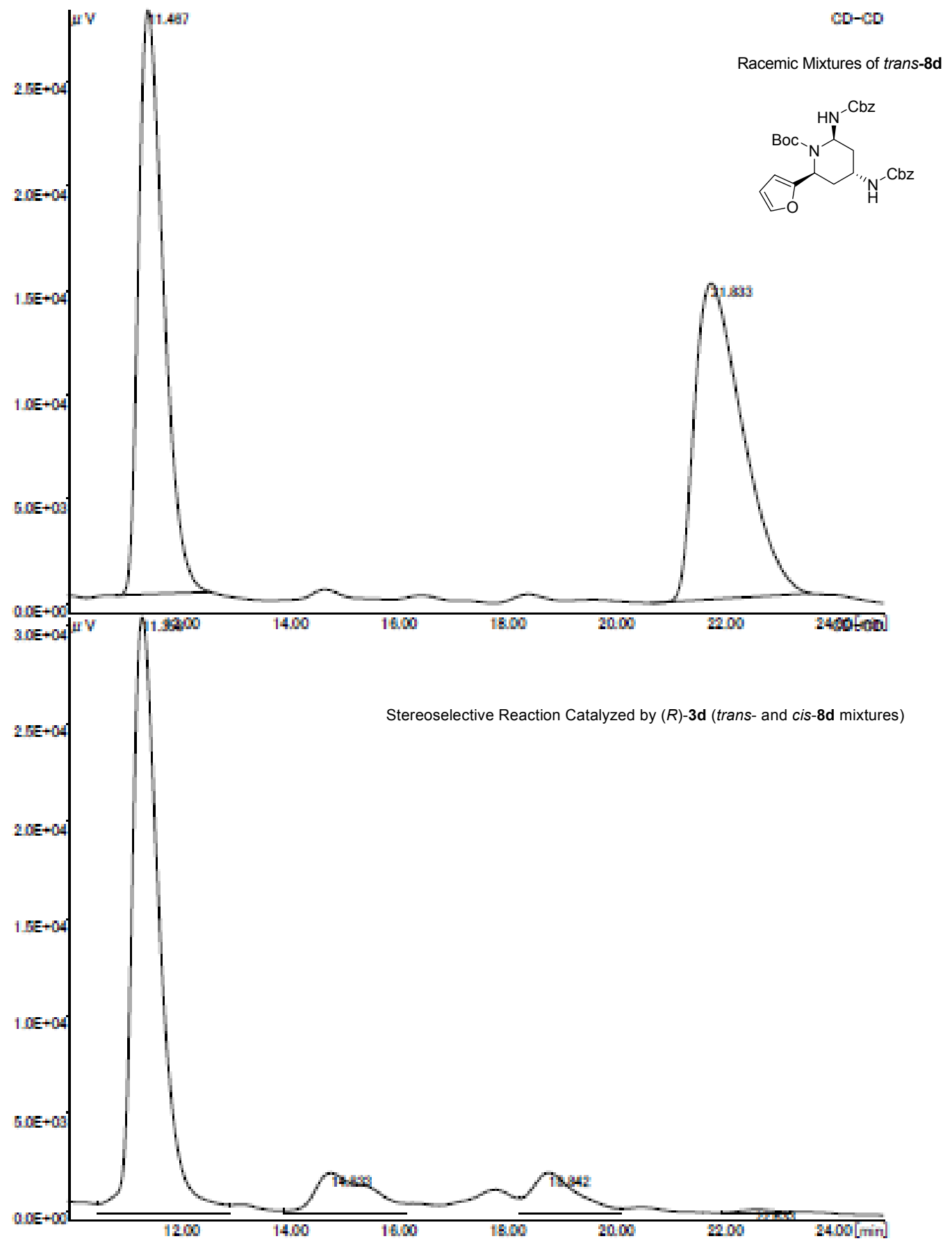




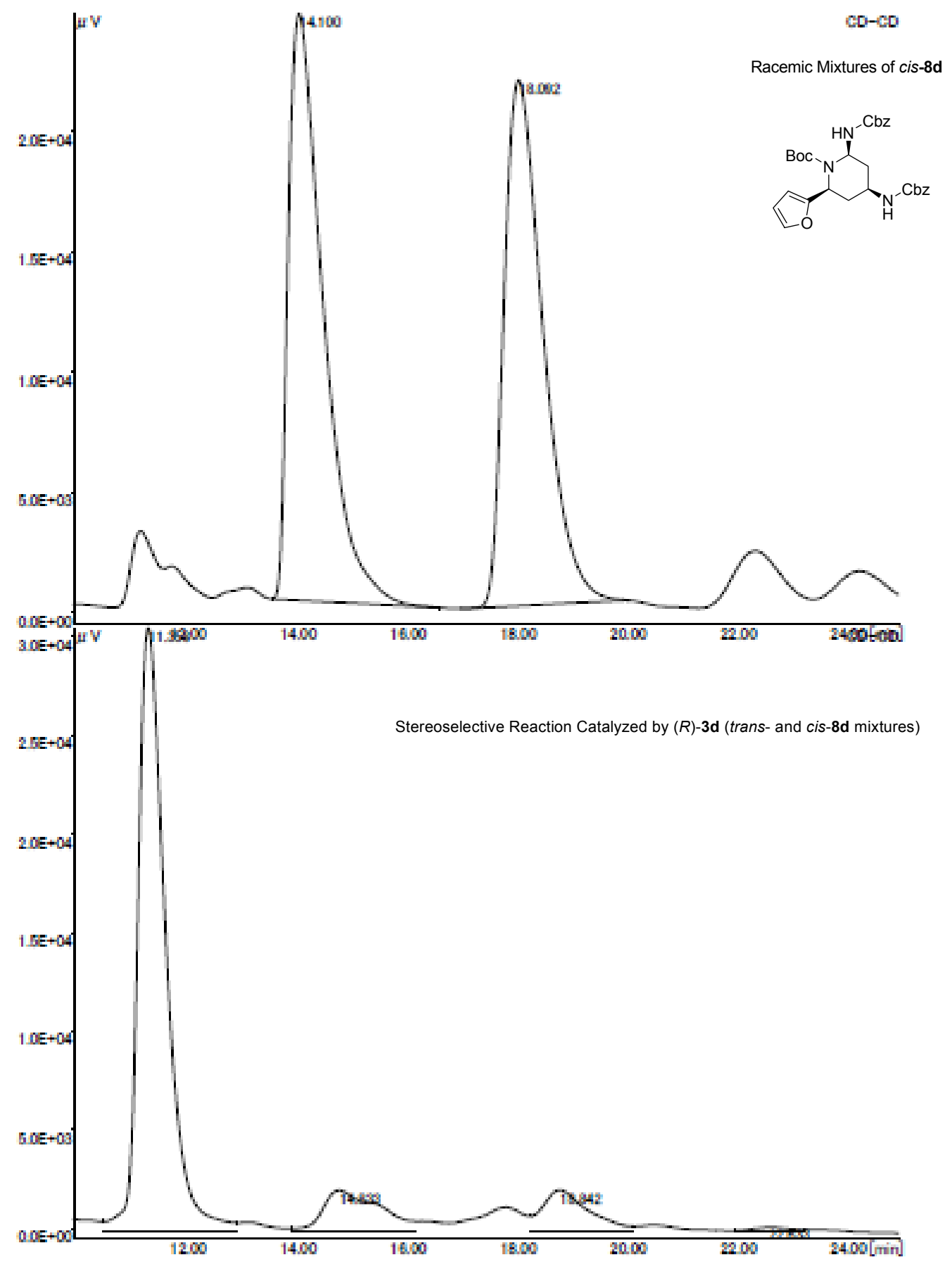



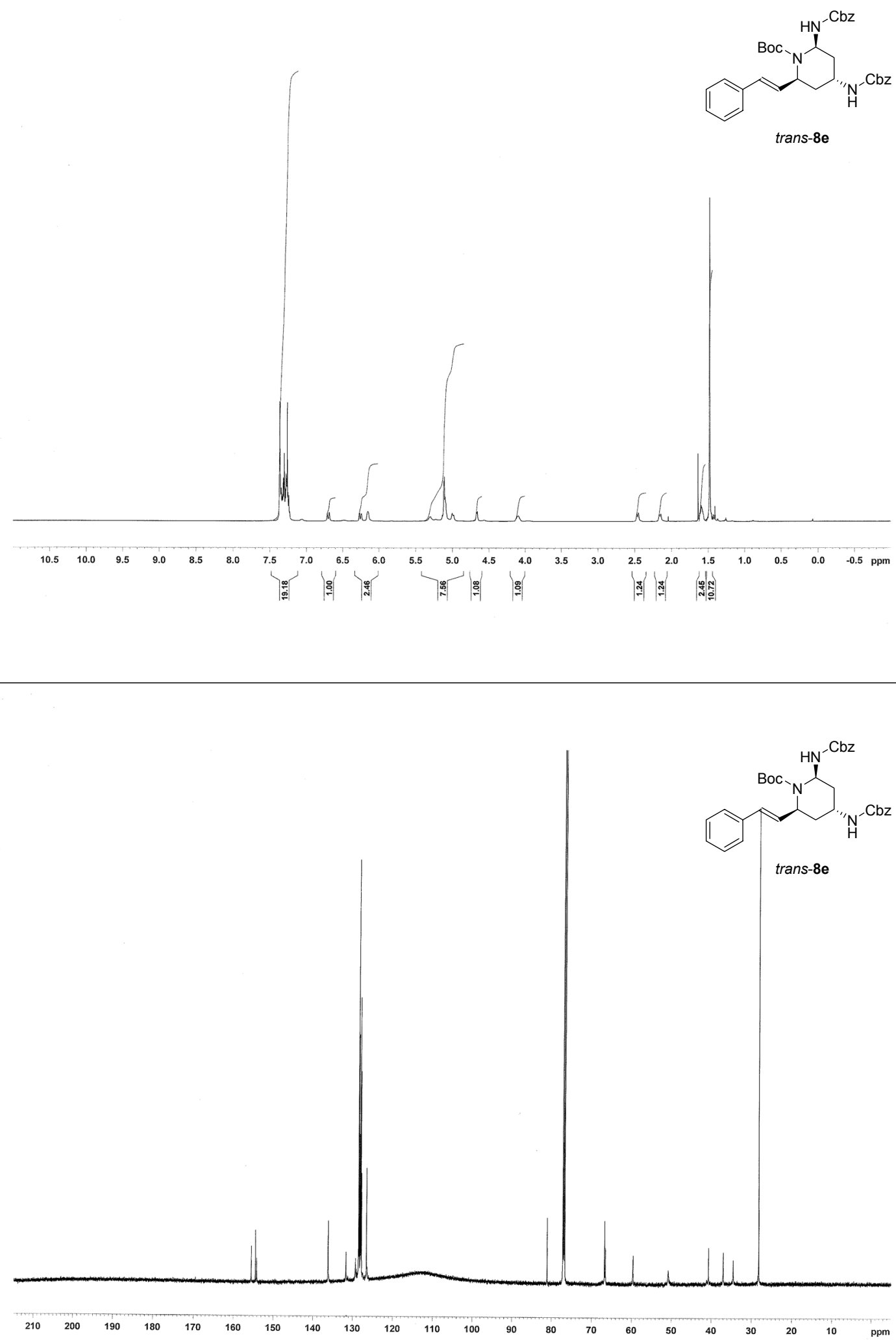

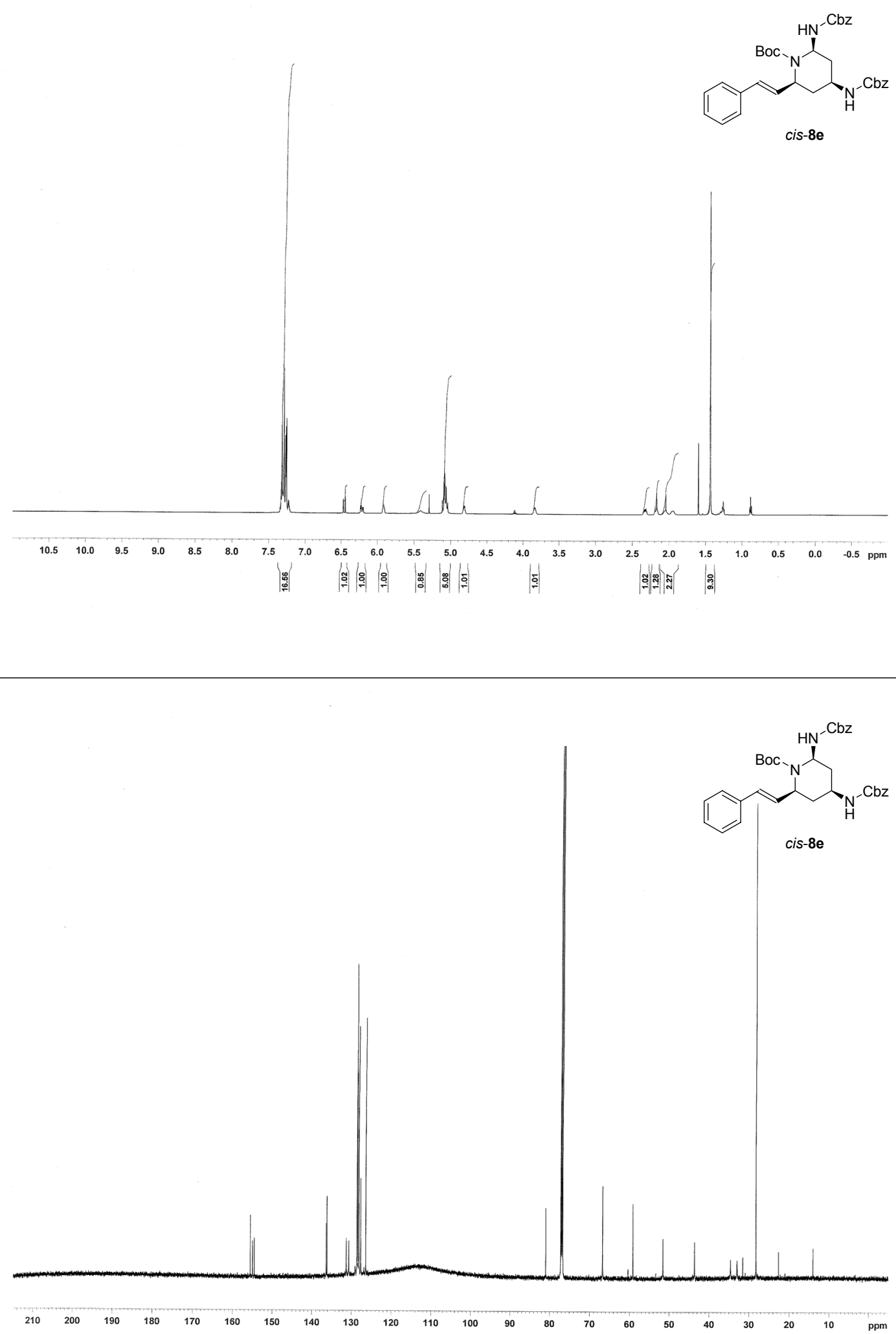


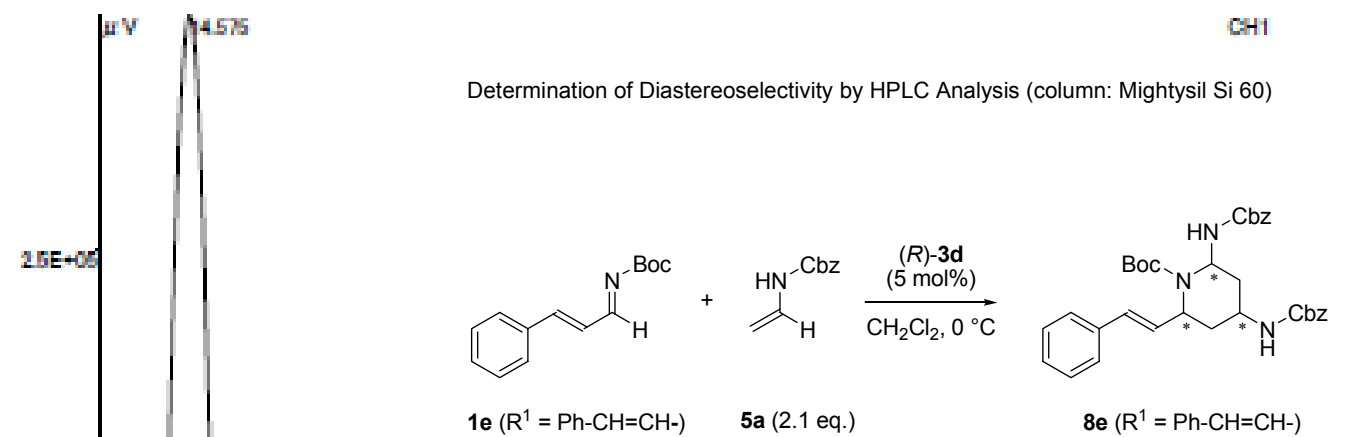

1e $\left(\mathrm{R}^{1}=\mathrm{Ph}-\mathrm{CH}=\mathrm{CH}-\right) \quad$ 5a $(2.1$ eq. $) \quad$ 8e $\left(\mathrm{R}^{1}=\mathrm{Ph}-\mathrm{CH}=\mathrm{CH}-\right)$ 


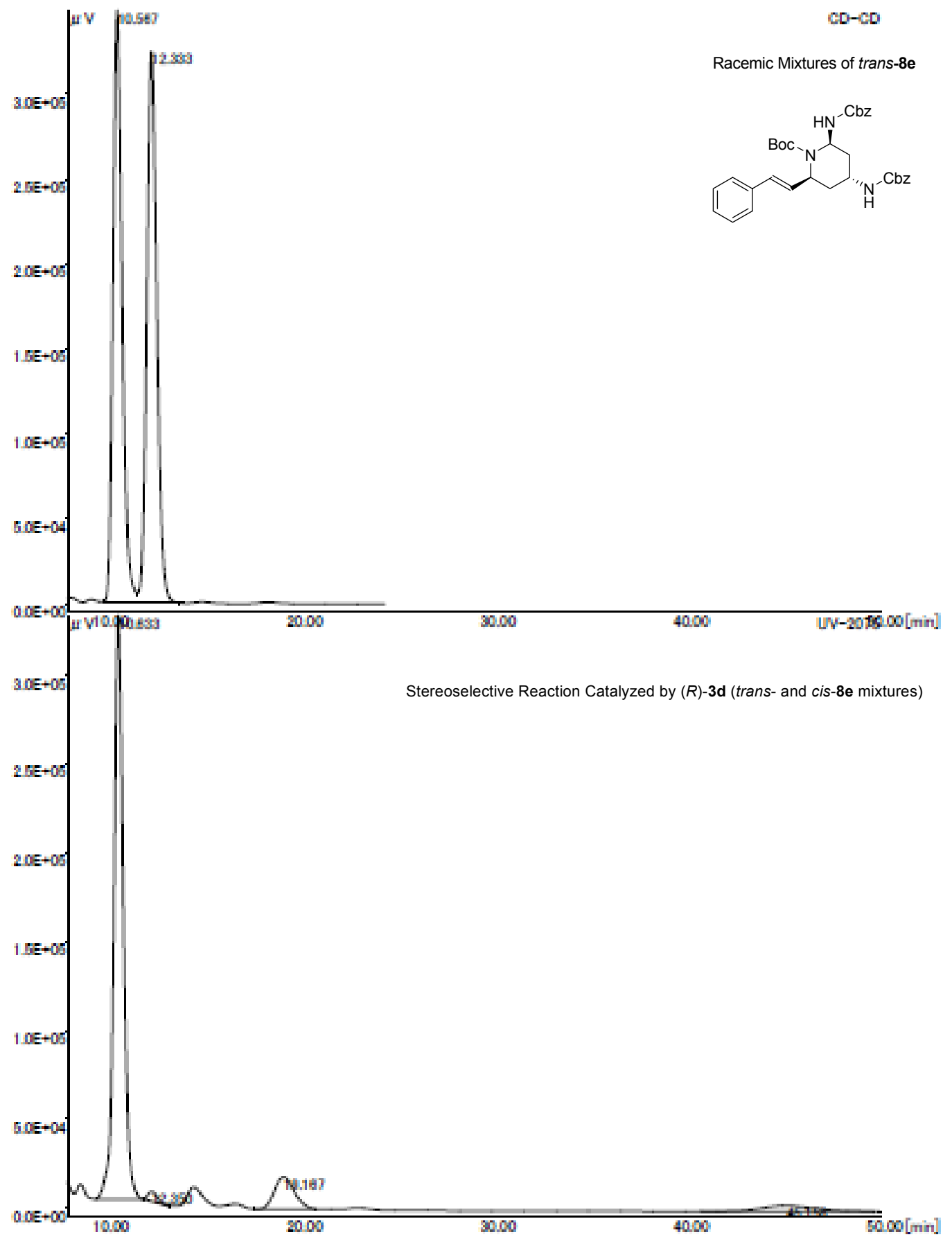




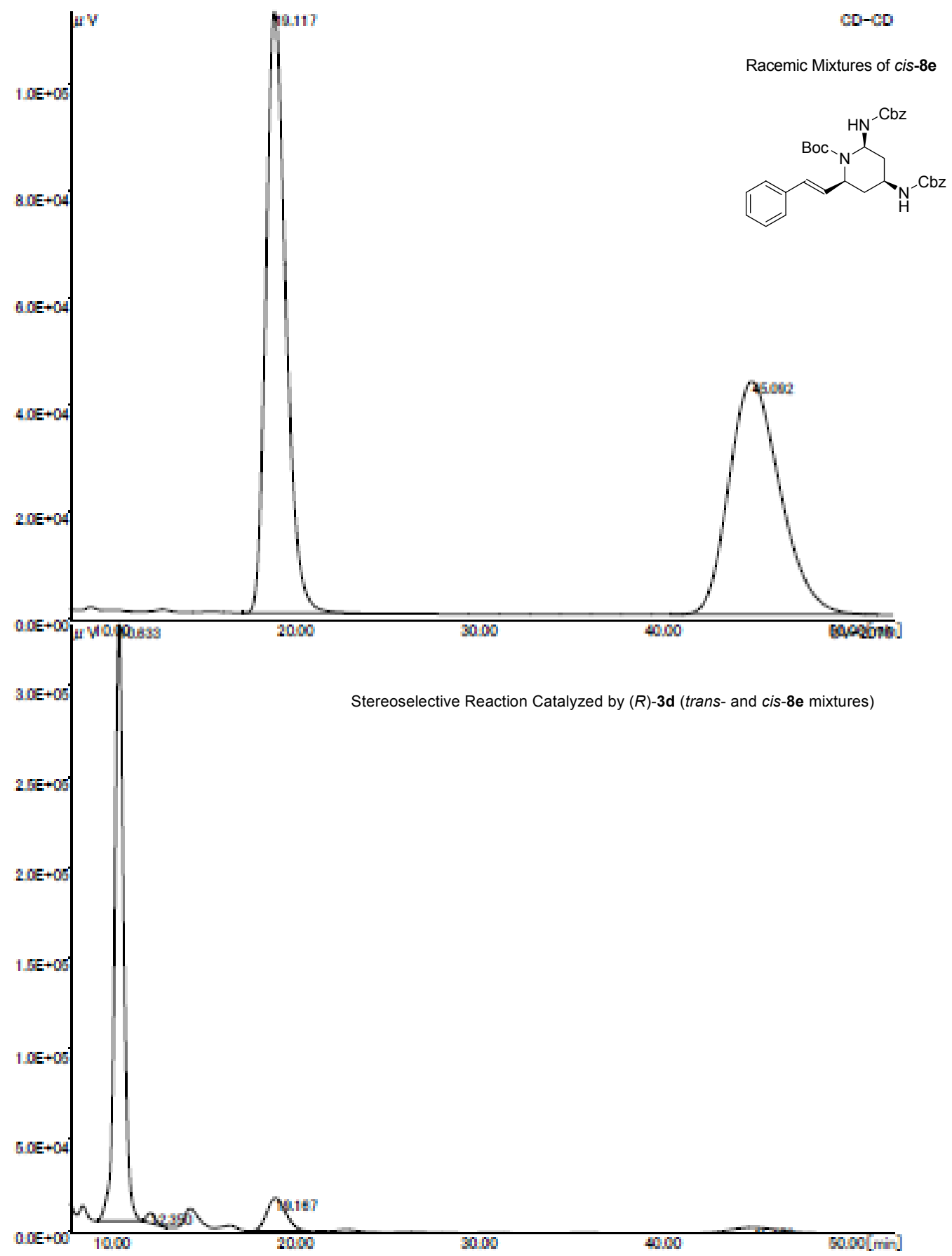




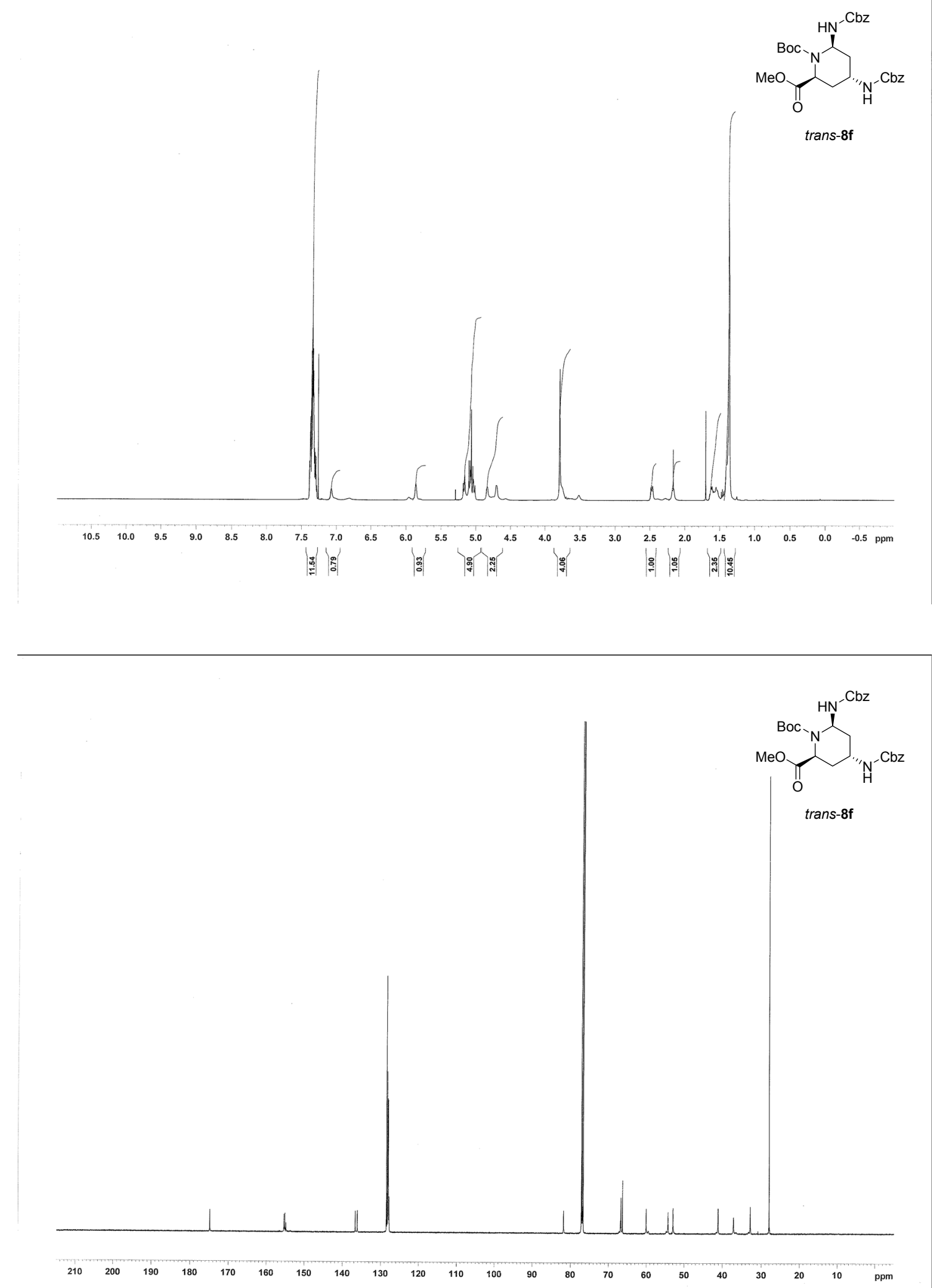




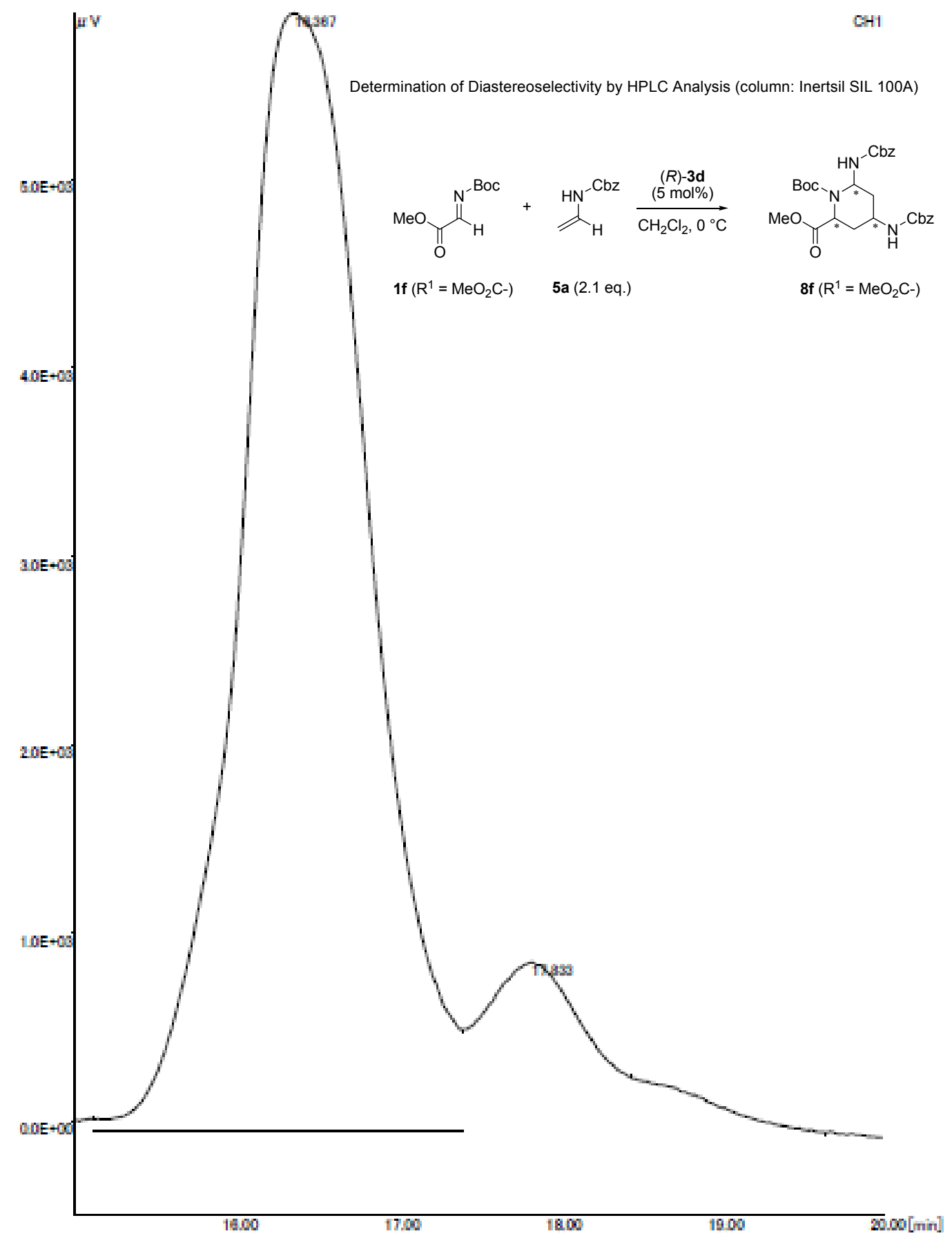




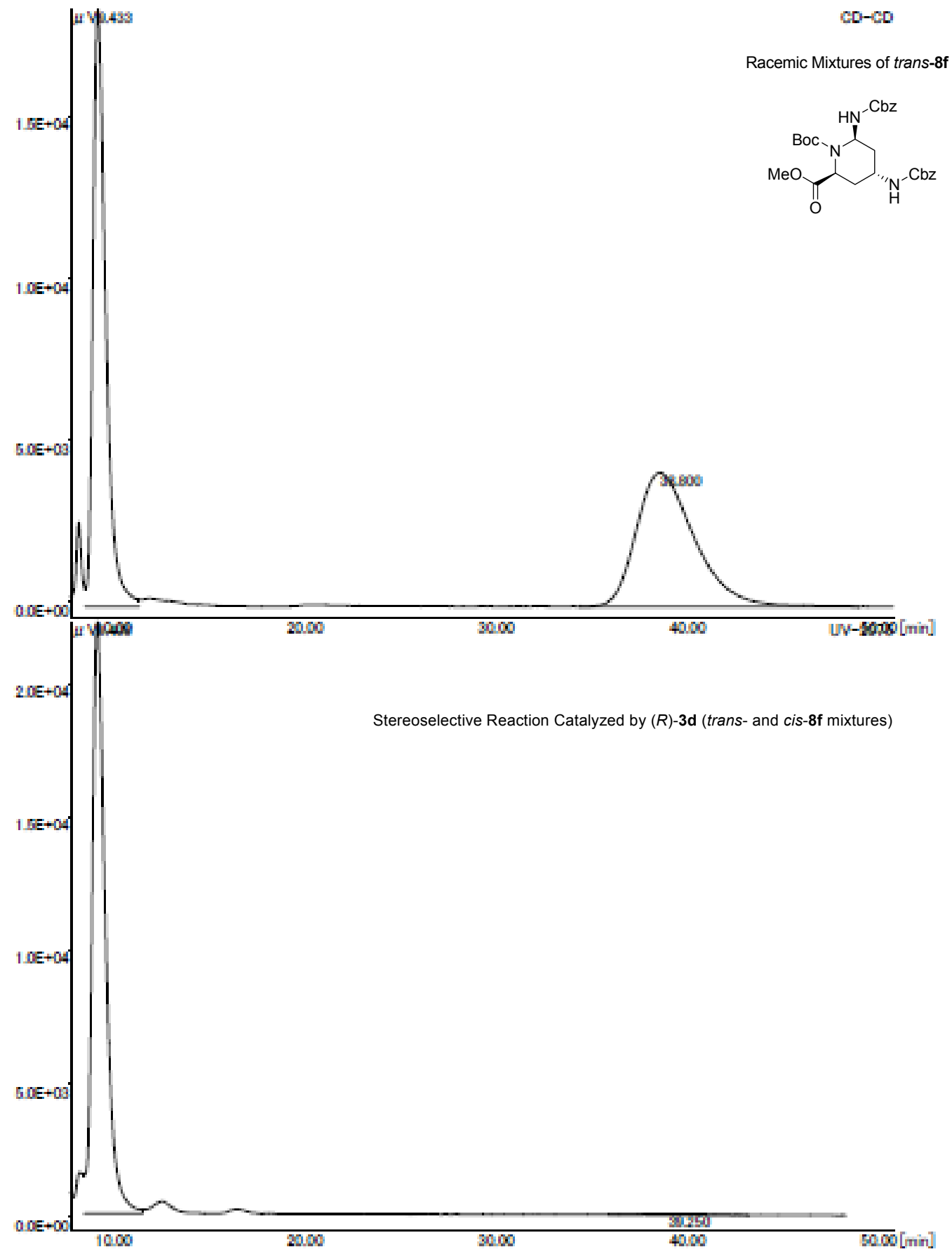



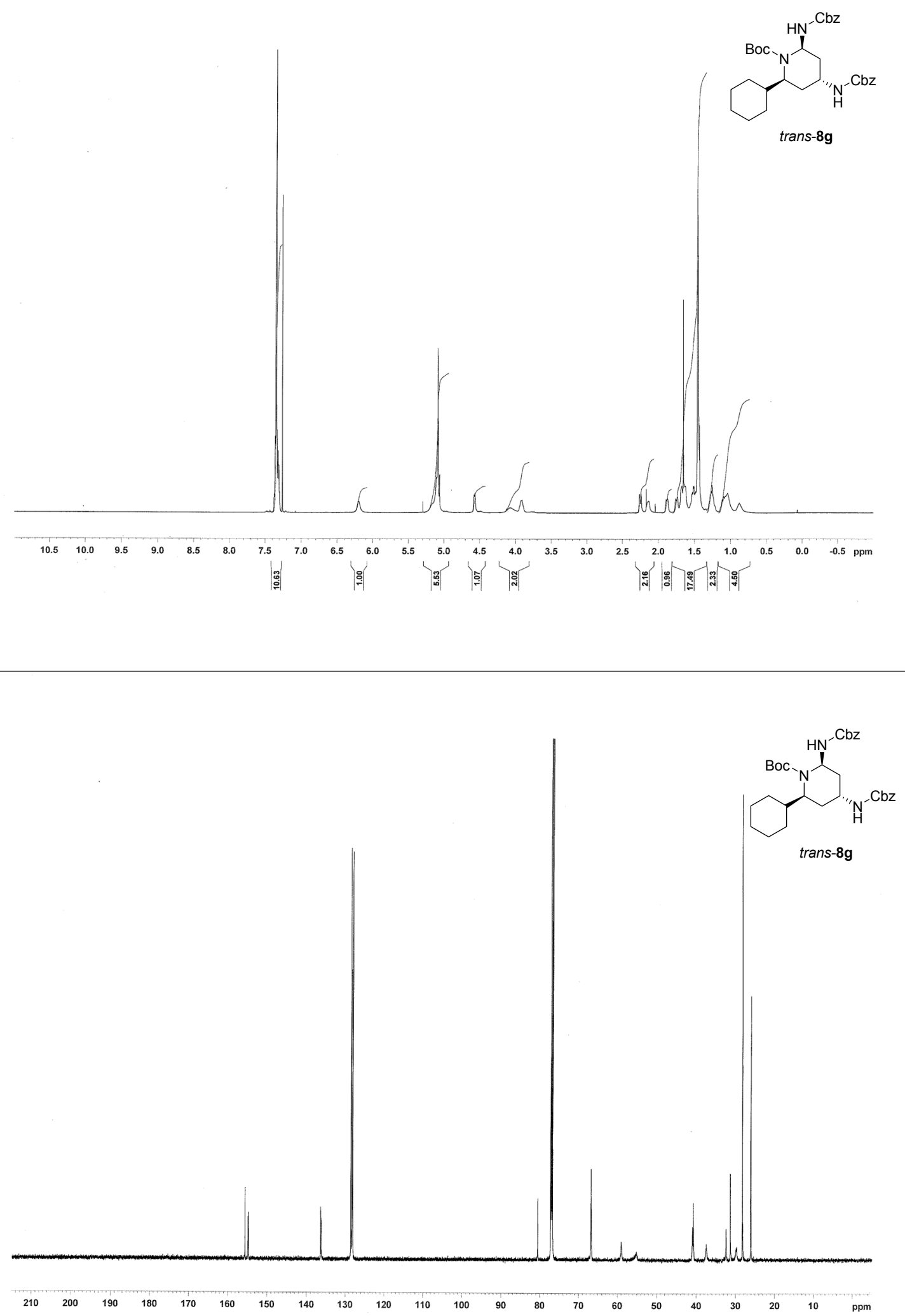

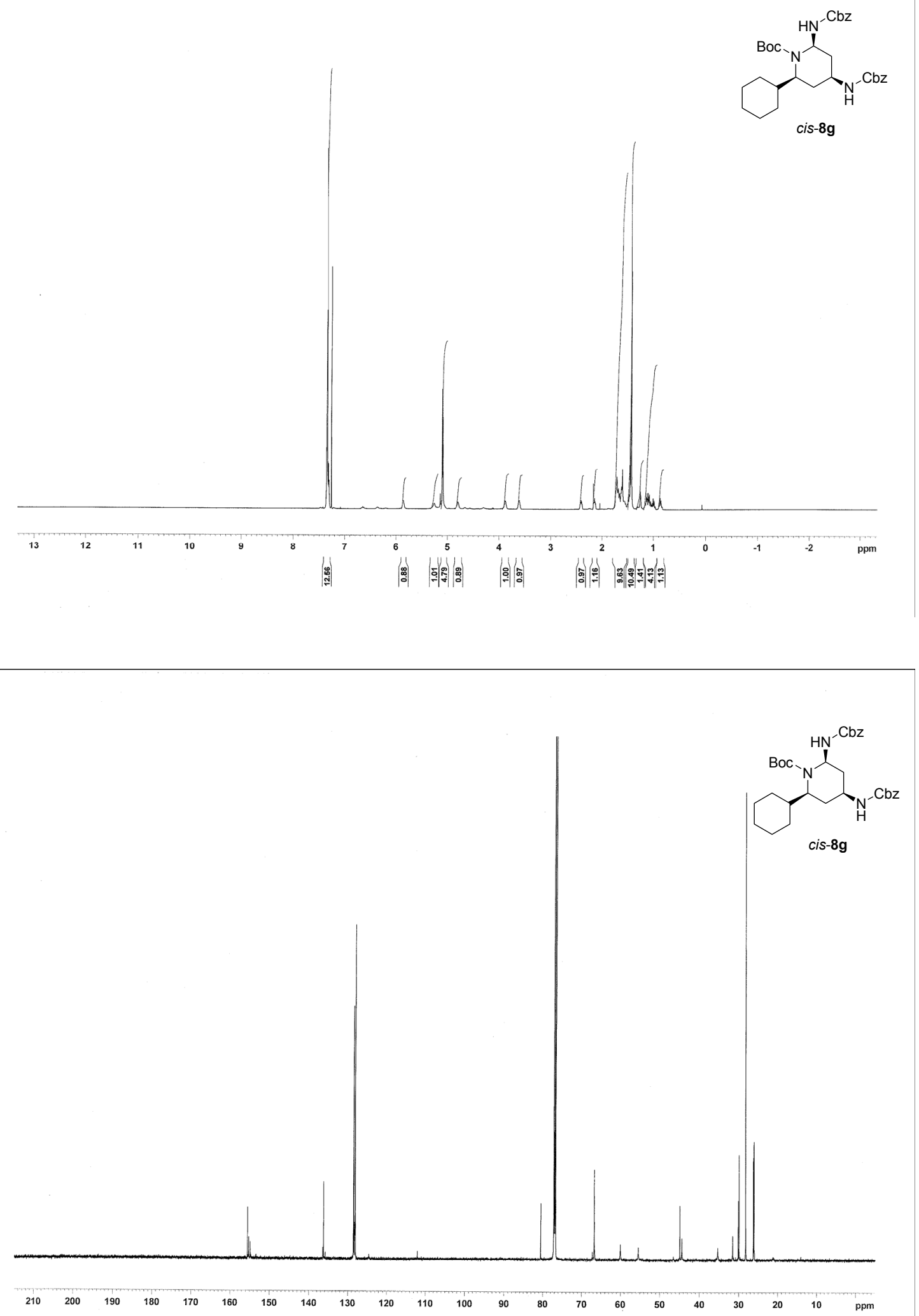


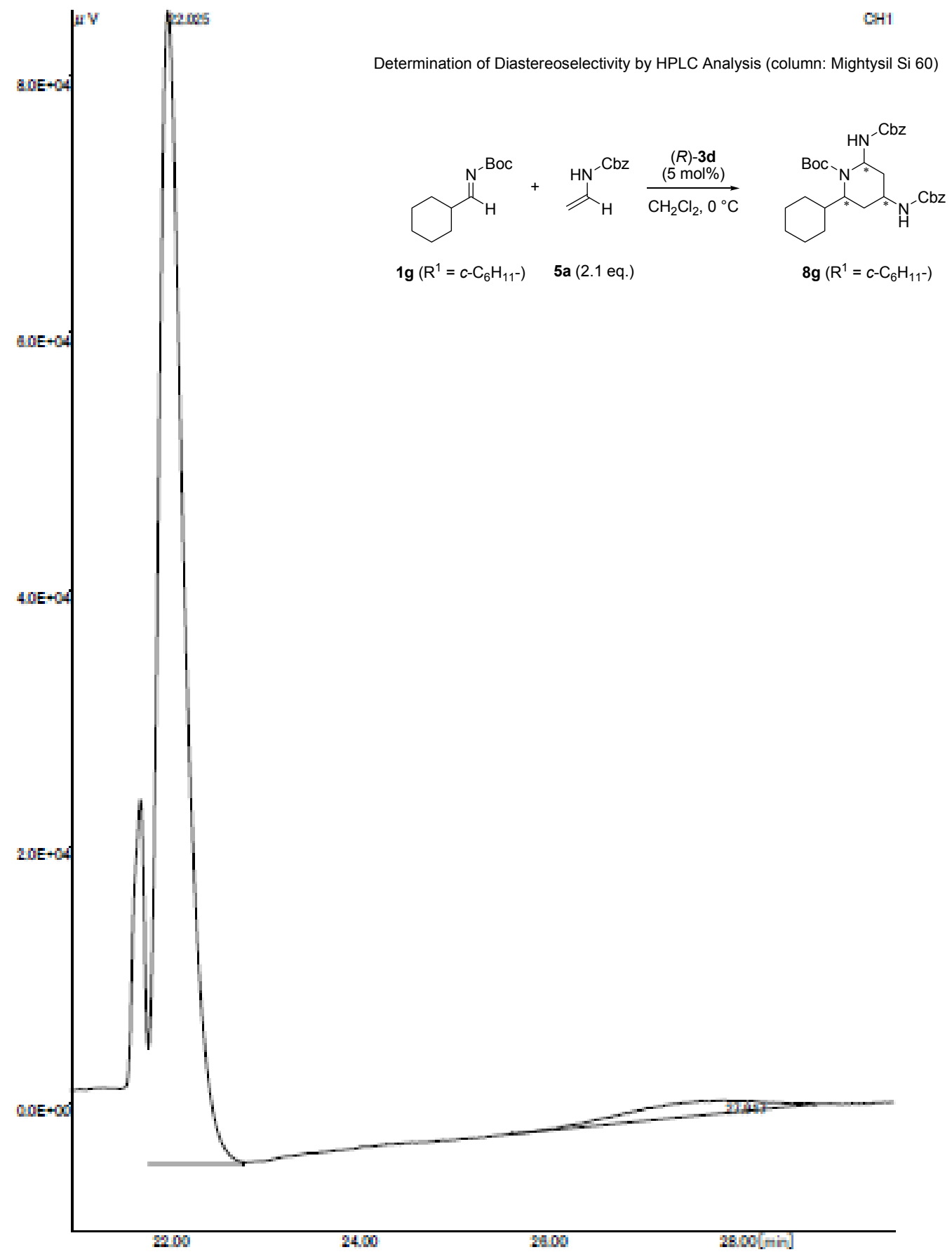




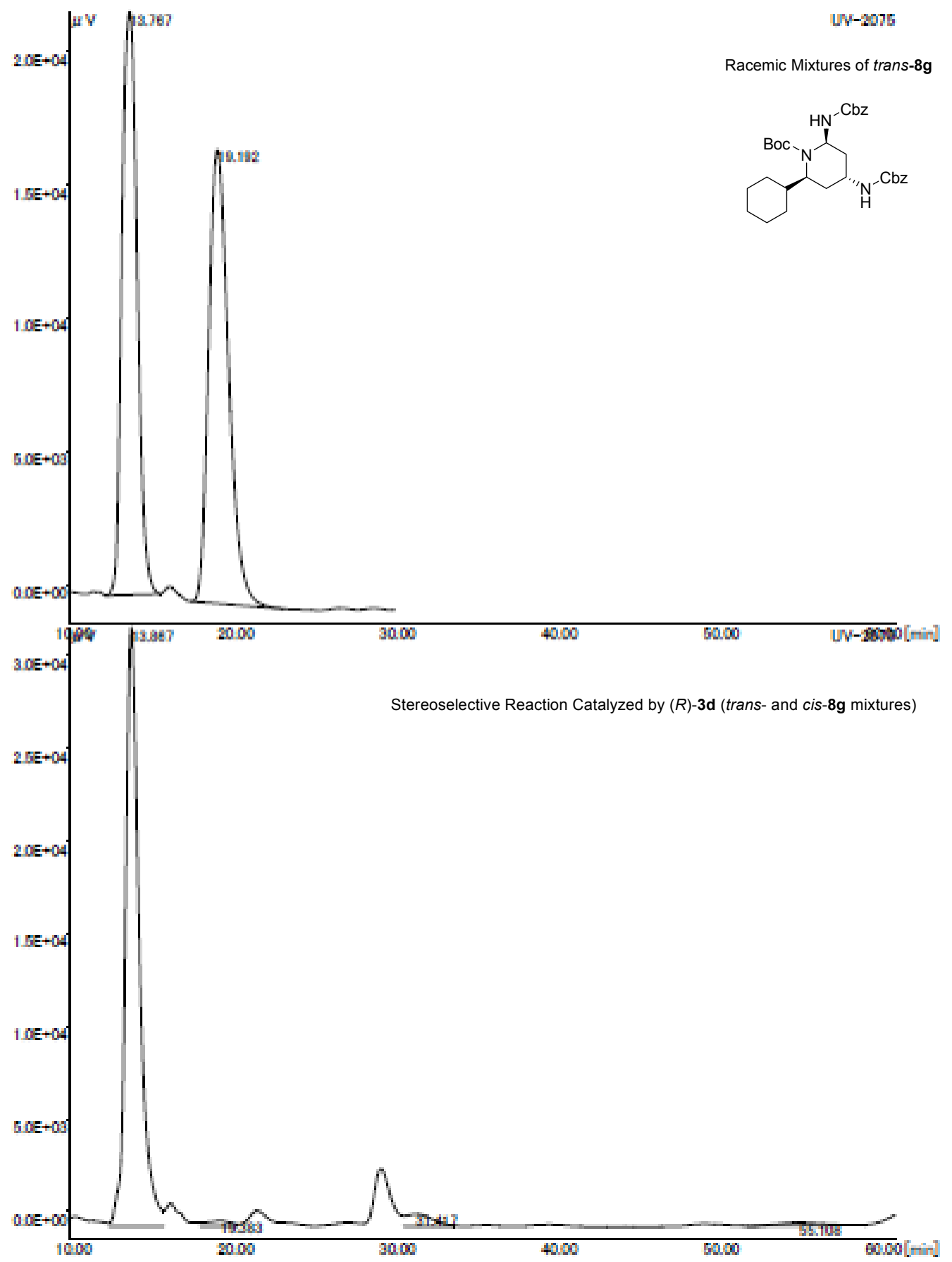




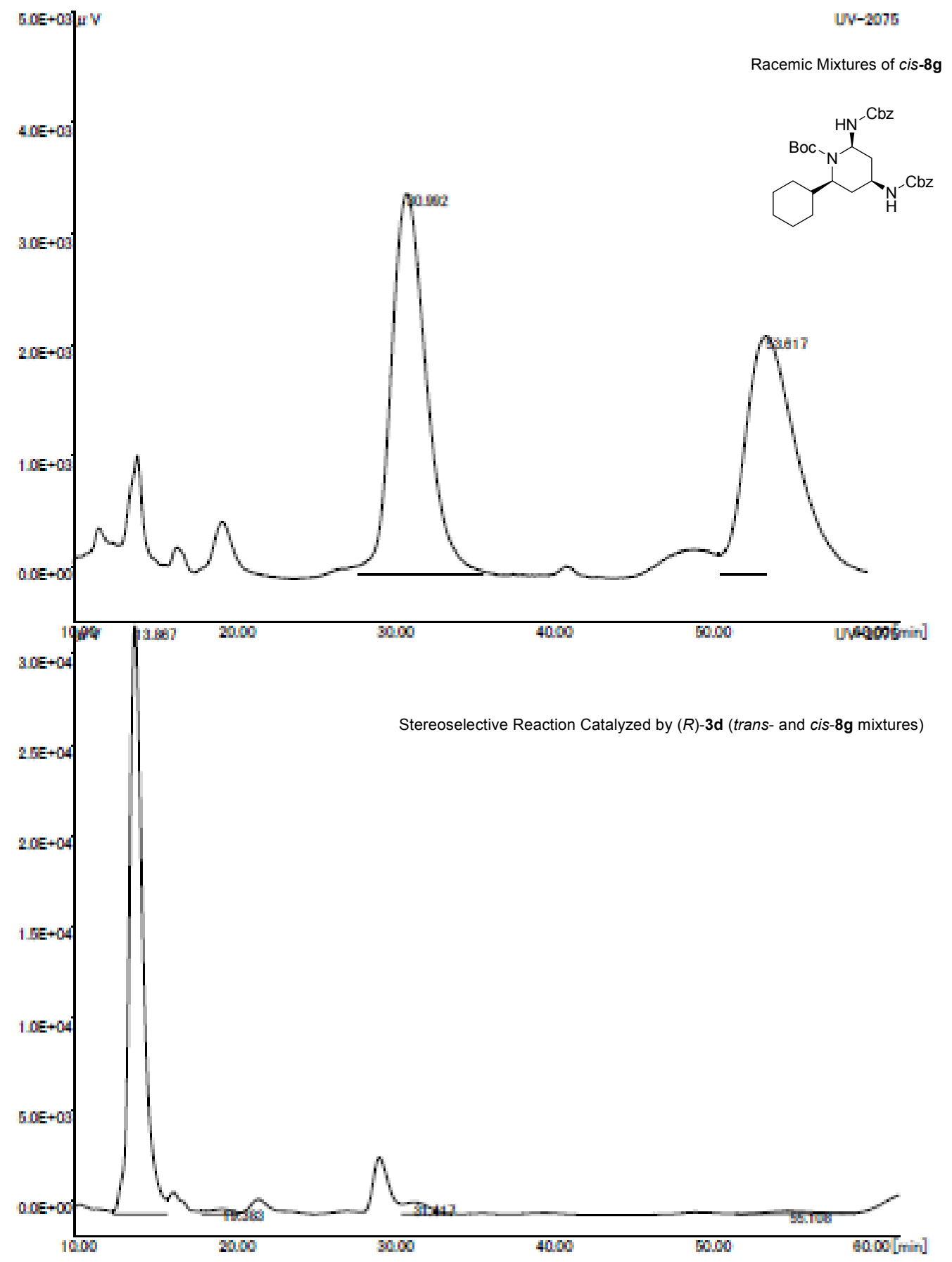

